\title{
Research Article \\ Scattering of Electromagnetic Plane Waves from a Coated PEMC Circular Cylinder Placed under PEC Wide Double Wedge
}

\author{
Muhammad Naveed, ${ }^{1}$ Shakeel Ahmed, ${ }^{2}$ and Qaisar Abbas Naqvi ${ }^{2}$ \\ ${ }^{1}$ National University of Sciences and Technology (NUST), Islamabad 44000, Pakistan \\ ${ }^{2}$ Department of Electronics, Quaid-i-Azam University, Islamabad 45320, Pakistan
}

Correspondence should be addressed to Qaisar Abbas Naqvi, nqaisar@yahoo.com

Received 30 July 2010; Accepted 27 November 2010

Academic Editor: Saad A. Ragab

Copyright (C) 2010 Muhammad Naveed et al. This is an open access article distributed under the Creative Commons Attribution License, which permits unrestricted use, distribution, and reproduction in any medium, provided the original work is properly cited.

Scattering of electromagnetic plane waves from a coated perfect electromagnetic conductor (PEMC) circular cylinder placed under perfect electric conducting (PEC) wide double wedge is presented. It is assumed that the distance between the two wedges is large as compared to the wavelength. Therefore, the field at an observation point can be considered to be composed of the incident field plus a response field from each of the edges of double wedge and the cylinder. PEMC cylinder is taken to be infinite along its axis and has been coated with a double positive (DPS) or double negative (DNG) material. The transmission coefficient and diffraction pattern of PEC wide double wedge in the presence of both coated and uncoated PEMC cylinder are studied. Results of special cases for PEMC cylinder, compared with the published work, are found to be in fairly good agreement. The techniques of Clemmow, and Karp and Russek have been used to investigate the transmission coefficient and diffraction pattern of the double wedge in the presence of both coated and un-coated PEMC circular cylinder.

\section{Introduction}

Scattering from multiple objects has been investigated by many researchers [1-6]. A possible technique is to use fictitious line sources, located according to the geometry of each scatterers. This technique was used by Clemmow [7], Karp and Russek [8] for the diffraction of electromagnetic plane waves by a wide slit. Elsherbeni and Hamid $[9,10]$ further extended it for wide double wedge and perfect electric conductor (PEC) cylinder. In this paper the same technique has been applied to perfect electromagnetic conductor (PEMC) circular cylinder coated with homogeneous, isotropic, and linear material placed under PEC wide double wedge. 
The concept of PEMC as the generalization of (PEC) and a perfect magnetic conductor (PMC) has been studied quite recently by Lindell and Sihvola [11]. It has attracted the attention of many researchers [12-19]. The PEMC boundary conditions are of the general form

$$
\vec{n} \times(\vec{H}+M \vec{E})=0 \quad \vec{n} \cdot(\vec{D}-M \vec{B})=0
$$

where $M$ denotes the admittance of the PEMC boundary. Here, PMC corresponds to $M=0$, while PEC corresponds to $M= \pm \infty$. In the recent years, there has been an increased interest in different classes of materials called meta materials like Double-Negative (DNG), Double Positive (DPS), Epsilon Negative (ENG) and Mu Negative (MNG). Veselago [20] characterized these mediums by negative real part of the permitivity as well as permeability. Scattering of electromagnetic plane waves by a conducting cylinder coated with meta material is investigated by Shen and Li [21] and Ahmed and Naqvi [22-26].

The electromagnetic scattering from an infinite PEMC cylinder coated with homogeneous, isotropic and linear DPS or DNG material placed under a PEC wide double wedge is studied. The known solutions for the scattered field by an isolated PEC wedge and an isolated coated PEMC cylinder are utilized. It is assumed that the field at any point is composed of the incident field and a response field from each of the double wedges and the cylinder. The response field consists of scattered field by the three scatterers due to the original plane wave plus an interaction field which will be represented by the three fictitious line sources located at the wedge edges and at the cylinder. The time dependence is assumed to be $\exp (j \omega t)$ and it is suppressed throughout the analysis.

\section{Formulation of the Problem}

For a single wedge, the two half planes of the wedge can be defined as $\phi=0$ and $\phi=2 \Phi$. The geometry of the problem is shown in Figure 1(a) where two parallel wedges loaded with coated PEMC cylinder is shown. The radius of the inner cylinder is $a$ and that of coated cylinder is $b$. The problem is two-dimensional since all the fields are uniform in the $z$-direction. By considering an E-polarized plane wave incident on an isolated PEC wedge and isolated PEMC cylinder, the known results of scattered field are presented in this section. The transmission coefficients for both coated and Uncoated cylinder are given. Figure 1(b) shows the geometry containing an Uncoated PEMC cylinder placed under PEC wide double wedge.

\subsection{PEC Wedge Excited by a Plane Wave}

For the plane wave incident on the edge of the wedge at an angle $\phi_{0}$ with respect to the negative $x$-axis the incident field is given as

$$
\left(\begin{array}{c}
E_{z}^{i} \\
H_{z}^{i}
\end{array}\right)=\left(\begin{array}{c}
E_{0} \\
H_{0}
\end{array}\right) \exp \left[j k\left(x \cos \phi_{0}+y \sin \phi_{0}\right)\right]
$$


The uniform expression for the field diffracted from a PEC wedge has the form [6]

$$
\left(\begin{array}{c}
E_{z}^{d} \\
H_{z}^{d}
\end{array}\right)=\frac{\exp [-j(k \rho)]}{\sqrt{\rho}} D_{h}^{s}\left(\rho, \phi, \phi_{0} ; n\right)\left(\begin{array}{c}
E_{z}^{i} \\
H_{z}^{i}
\end{array}\right) .
$$

The diffraction coefficient for the PEC wedge is

$$
\begin{aligned}
D_{h}^{s}\left(\rho, \phi, \phi_{0} ; n\right)=-\sqrt{\rho}\{ & -\operatorname{sgn}\left(\sin \left(\frac{\pi+\phi-\phi_{0}}{2 n}\right)\right) \cos \left(\frac{\pi+\phi-\phi_{0}}{2 n}\right) \\
& \times F\left[\sqrt{2 k \rho n}\left|\sin \left(\frac{\pi+\phi-\phi_{0}}{2 n}\right)\right|\right] \\
& -\operatorname{sgn}\left(\sin \left(\frac{\pi-\left(\phi-\phi_{0}\right)}{2 n}\right)\right) \cos \left(\frac{\pi-\left(\phi-\phi_{0}\right)}{2 n}\right) \\
& \times F\left[\sqrt{2 k \rho n}\left|\sin \left(\frac{\pi-\left(\phi-\phi_{0}\right)}{2 n}\right)\right|\right] \\
& \pm \operatorname{sgn}\left(\sin \left(\frac{\pi+\phi+\phi_{0}}{2 n}\right)\right) \cos \left(\frac{\pi+\phi+\phi_{0}}{2 n}\right) \\
& \times F\left[\sqrt{2 k \rho n}\left|\sin \left(\frac{\pi+\phi+\phi_{0}}{2 n}\right)\right|\right] \\
& \pm \operatorname{sgn}\left(\sin \left(\frac{\pi-\left(\phi+\phi_{0}\right)}{2 n}\right)\right) \cos \left(\frac{\pi-\left(\phi+\phi_{0}\right)}{2 n}\right) \\
& \left.\times F\left[\sqrt{2 k \rho n}\left|\sin \left(\frac{\pi-\left(\phi+\phi_{0}\right)}{2 n}\right)\right|\right]\right\}
\end{aligned}
$$

$D_{s}$ and $D_{h}$ are the diffraction coefficients of $E$ - and $H$-polarization, respectively, and $n=$ $2 \Phi / \pi$. Function $\operatorname{sgn}(\cdot)$ is the signum function whereas $F(\cdot)$ is the Fresnel integral defined as

$$
F(x)=\frac{1}{\pi} \exp \left(j x^{2}+j \frac{\pi}{4}\right) \int_{x}^{\infty} \exp \left(-j \mu^{2}\right) d \mu
$$

It is assumed that point of observation is far from the edge of the wedge. For large argument approximation, Fresnel integral simplifies as

$$
F(x) \approx \frac{1}{2 \sqrt{\pi} x} \exp \left[-j \frac{\pi}{4}\right]
$$




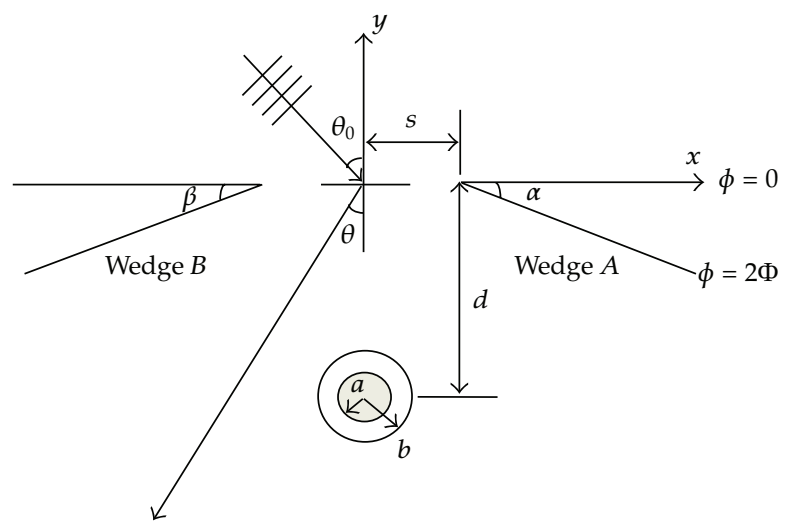

(a)

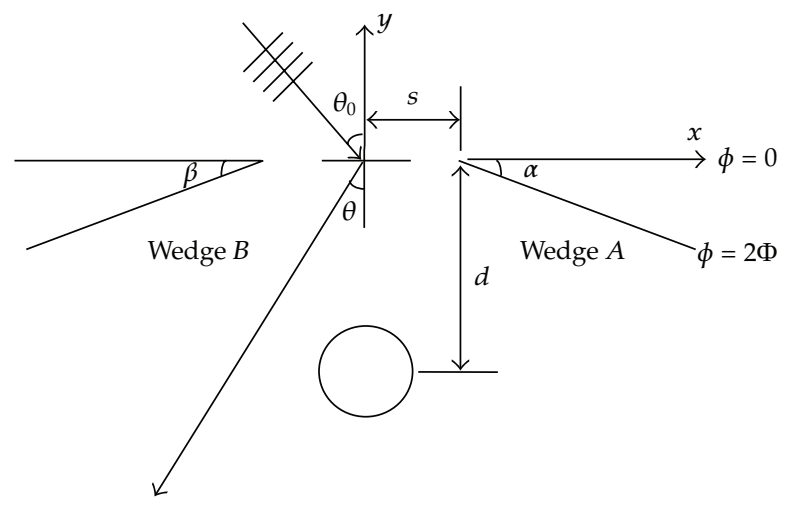

(b)

Figure 1: (a) Geometry of the Problem-Coated PEMC cylinder placed under PEC wide double wedge. (b) Uncoated PEMC cylinder placed under PEC wide double wedge.

Hence diffraction coefficient for E-polarized plane wave, incident on the edge of the wedge simplifies to

$$
D\left(\phi, \phi_{0}, n\right) \approx \frac{\sin (\pi / n)}{\pi n}\left\{\left[\cos \left(\frac{\pi}{n}\right)-\cos \left(\frac{\phi-\phi_{0}}{n}\right)\right]^{-1}-\left[\cos \left(\frac{\pi}{n}\right)-\cos \left(\frac{\phi+\phi_{0}}{n}\right)\right]^{-1}\right\} .
$$

The angles between the incident and diffracted rays and normal to the screen are $\phi$ and $\phi_{0}$, respectively.

\subsection{Circular Cylinder Excited by a Plane Wave}

A circular cylinder is defined by the surface $\rho=a$, while its axis coincides with the $z$-axis. The radiated fields due to plane wave incident on a circular cylinder [27] are

$$
E_{p}^{C}=\frac{\pi}{2 j} H_{0}^{(2)}(k \rho) G_{p}\left(\phi, \phi_{0}, a\right),
$$


where

$$
G_{p}\left(\phi, \phi_{0}, a\right)=-\frac{2 j}{\pi} \sum_{n=0}^{\infty} \epsilon_{n}(-1)^{n} T_{n} \cos \left[n\left(\phi-\phi_{0}\right)\right]
$$

where the Neumann number $\epsilon_{n}=1$ for $n=0$ and 2 for $n>0$. In (2.8), $T_{n}$ is the transmission coefficient. Its values for both co and cross-polarized components of Uncoated PEMC cylinder $[18]$ is

$$
T_{n}= \begin{cases}\frac{H_{n}^{(2)}(k a) J_{n}^{\prime}(k a)+M^{2} \eta_{0}^{2} J_{n}(k a) H_{n}^{(2))^{\prime}}(k a)}{\left(1+M^{2} \eta_{0}^{2}\right) H_{n}^{(2)}(k a) H_{n}^{(2)^{\prime}}(k a)}, & \text { Copolarized } \\ \frac{2 M \eta_{0}}{\pi k a\left(1+M^{2} \eta_{0}^{2}\right) H_{n}^{(2)}(k a) H_{n}^{(2)^{\prime}}(k a)}, & \text { Cross polarized }\end{cases}
$$

whereas the transmission coefficient for coated PEMC cylinder [22] is

$$
\begin{gathered}
T_{\mathrm{co}}=\frac{\left(J_{n}^{\prime}\left(k_{0} b\right) / \eta_{0}\right)(A)-\left(J_{n}\left(k_{0} b\right) / \eta_{1}\right)(B)}{\left(H_{n}^{(2)}\left(k_{0} b\right) / \eta_{1}\right)(B)-\left(H_{n}^{(2)^{\prime}}\left(k_{0} b\right) / \eta_{0}\right)(A)}, \\
T_{\text {cross }}=j M \eta_{1}\left[H_{n}^{(1)}\left(k_{1} a\right)-\frac{H_{n}^{(2)}\left(k_{1} a\right) H_{n}^{(1)^{\prime}}\left(k_{1} a\right)}{H_{n}^{(2)^{\prime}}\left(k_{1} a\right)}\right]\left[\frac{J_{n}\left(k_{0} b\right) H_{n}^{(2)^{\prime}}\left(k_{0} b\right)-J_{n}^{\prime}\left(k_{0} b\right) H_{n}^{(2)}\left(k_{0} b\right)}{\eta_{0} H_{n}^{(2)}\left(k_{0} b\right)(B)-H_{n}^{(2))^{\prime}}\left(k_{0} b\right)(A)}\right],
\end{gathered}
$$

where

$$
\begin{aligned}
& A= {\left[\left(\frac{a c}{b}+d\right)-j M \eta_{1} \frac{H_{n}^{(2)}\left(k_{1} a\right)}{H_{n}^{(2)^{\prime}}\left(k_{1} a\right)}\left(\frac{e a}{b}+f\right)\right]\left[H_{n}^{(1)}\left(k_{1} b\right)-\frac{H_{n}^{(2)}\left(k_{1} b\right) H_{n}^{(1)^{\prime}}\left(k_{1} a\right)}{H_{n}^{(2)^{\prime}}\left(k_{1} a\right)}\right] } \\
&+j M \eta_{1} \frac{H_{n}^{(2)}\left(k_{1} b\right)}{H_{n}^{(2)^{\prime}}\left(k_{1} a\right)}\left(\frac{e a}{b}+f\right)\left[H_{n}^{(1)}\left(k_{1} a\right)-\frac{H_{n}^{(2)}\left(k_{1} a\right) H_{n}^{(1)^{\prime}}\left(k_{1} a\right)}{H_{n}^{(2)^{\prime}}\left(k_{1} a\right)}\right], \\
& B= {\left[H_{n}^{(1)^{\prime}}\left(k_{1} b\right)-\frac{H_{n}^{(2)^{\prime}}\left(k_{1} b\right) H_{n}^{(1)^{\prime}}\left(k_{1} a\right)}{H_{n}^{(2)^{\prime}}\left(k_{1} a\right)}\right]\left[\left(\frac{a c}{b}+d\right)-j M \eta_{1} \frac{H_{n}^{(2)}\left(k_{1} a\right)}{H_{n}^{(2)^{\prime}}\left(k_{1} a\right)}\left(\frac{e a}{b}+f\right)\right] } \\
&+j M \eta_{1} \frac{H_{n}^{(2)^{\prime}}\left(k_{1} b\right)}{H_{n}^{(2)^{\prime}}\left(k_{1} a\right)}\left(\frac{e a}{b}+f\right)\left[H_{n}^{(1)}\left(k_{1} a\right)-\frac{H_{n}^{(2)}\left(k_{1} a\right) H_{n}^{(1)^{\prime}}\left(k_{1} a\right)}{H_{n}^{(2)^{\prime}}\left(k_{1} a\right)}\right], \\
& a=\frac{H_{n}^{(2)}\left(k_{0} b\right)}{\eta_{0}}-\frac{H_{n}^{(2)}\left(k_{1} b\right) H_{n}^{(2) \prime^{\prime}}\left(k_{0} b\right)}{\eta_{1} H_{n}^{(2)^{\prime}}\left(k_{1} b\right)},
\end{aligned}
$$




$$
\begin{aligned}
& b=\frac{1}{\eta_{1}}\left[H_{n}^{(1)}\left(k_{1} b\right)-\frac{H_{n}^{(2)}\left(k_{1} b\right) H_{n}^{(2)^{\prime}}\left(k_{1} b\right)}{H_{n}^{(2)^{\prime}}\left(k_{1} b\right)}\right], \\
& c=H_{n}^{(1)}\left(k_{1} a\right)-\frac{H_{n}^{(2)}\left(k_{1} a\right) H_{n}^{(1)^{\prime}}\left(k_{1} b\right)}{H_{n}^{(2)^{\prime}}\left(k_{1} b\right)}, \\
& d=\frac{H_{n}^{(2)}\left(k_{1} a\right) H_{n}^{(2)^{\prime}}\left(k_{0} b\right)}{H_{n}^{(2)^{\prime}}\left(k_{1} b\right)}, \\
& e=j M \eta_{1}\left[H_{n}^{(1)^{\prime}}\left(k_{1} a\right)-\frac{H_{n}^{(1)^{\prime}}\left(k_{1} b\right) H_{n}^{(2)^{\prime}}\left(k_{1} a\right)}{H_{n}^{(2)^{\prime}}\left(k_{1} b\right)}\right], \\
& f=j M \eta_{1} \frac{H_{n}^{(2)^{\prime}}\left(k_{0} b\right) H_{n}^{(2)^{\prime}}\left(k_{1} a\right)}{H_{n}^{(2)^{\prime}}\left(k_{1} b\right)} .
\end{aligned}
$$

In above equations $J_{n}(\cdot)$ is the Bessel function of order $n$ and $H_{n}(\cdot)$ is the Hankel function of second kind of order $n$. Primes indicate the derivative with respect to the whole argument.

\section{Cylindrical Wave Incident}

In this section, the known solutions due to a line source excitation for scattered fields from isolated PEC wedge and from coated PEMC cylinder are presented. The purpose is to get the interaction contribution from each of the two wedges and a coated PEMC cylinder using the known solutions and by incorporating the techniques used by Clemmow [7], and Karp and Russek [8].

\subsection{PEC Wedge Excited by a Cylindrical Wave}

The field of a line source in the presence of a conducting wedge whose edge is parallel to the source is well known. If the source is of unit amplitude and is located at $\left(\rho_{0}, \phi_{0}\right)$ parallel to the $z$-axis, its field in the absence of the wedge is given as

$$
E^{i}=\frac{\pi}{2 j} H_{0}^{(2)}(k R)
$$

where $R$ is the distance between the line source and the field point, $k$ is the wave number, and $H_{0}^{(2)}(\cdot)$ is the Hankel function of the second kind of order zero. The diffracted field in the presence of the wedge is given asymptotically in [28]. Here the asymptotic expression of the Hankel function is replaced by the Hankel function itself, therefore, the diffracted field has 
the appearance of cylindrical wave emanating from a line source located at the edge of the wedge expressed in the form

$$
E=\frac{\pi}{2 j} H_{0}^{(2)}(k \rho) F\left(\phi, \rho_{0}, \phi_{0}, n\right)
$$

where

$$
\begin{aligned}
F\left(\phi, \rho_{0}, \phi_{0}, n\right) \approx & \frac{H_{0}^{(2)}\left(k \rho_{0}\right) \exp [-j(\pi / 2)] \sin (\pi / n)}{\pi n} \\
& \times\left\{\left[\cos \left(\frac{\pi}{n}\right)-\cos \left(\frac{\phi-\phi_{0}}{n}\right)\right]^{-1}-\left[\cos \left(\frac{\pi}{n}\right)-\cos \left(\frac{\phi+\phi_{0}}{n}\right)\right]^{-1}\right\} .
\end{aligned}
$$

Here, again the angles between the incident and diffracted rays and normal to the screen are $\phi$ and $\phi_{0}$, respectively.

\subsection{PEMC-Coated Circular Cylinder Excited by a Cylindrical Wave}

The scattered field due to cylindrical wave incident on circular cylinder [27] is

$$
E_{l}^{C}=\frac{\pi}{2 j} H_{0}(k \rho) G_{l}\left(\phi, \rho_{0}, \phi_{0}, a\right)
$$

where

$$
G_{l}\left(\phi, \rho_{0}, \phi_{0}, a\right)=-\sum_{n=0}^{\infty} \epsilon_{n} j^{n} T_{n} H_{n}\left(k \rho_{0}\right) \cos \left[n\left(\phi-\phi_{0}\right)\right]
$$

where $T_{n}$ is the transmission coefficient. Its values for co and cross-polarized components of both Uncoated and coated PEMC cylinders are given by (2.9) and (2.10).

\section{Interaction Contributions of the Geometry}

There are two conducting wedges separated by a distance $2 s$, where $2 k s \gg 1$ and a coated PEMC circular cylinder of radius $b$ whose axis is parallel to the edges of two parallel wedges as shown in Figure 1(a). All the three bodies are considered to be illuminated by a plane wave of unit amplitude. The field at any point is considered to be composed of the incident field plus a response field from each of the two wedges and the cylinder. The response field consists of scattered field by the three scatterers due to the original plane wave (the noninteraction field) plus an interaction field which will be represented by three fictitious line sources located at the wedge edges and at the cylinder in order to take into account multiple interaction between three objects. Consider, for example, edge of wedge $A$ which is excited by direct plane wave plus line source fields of edge $B$ (the second wedge)and edge $C$ (the cylinder axis). The interaction can be conveniently expressed in terms of the response of edge $A$ to the line source at the opposite edge and at the cylinder axis. 


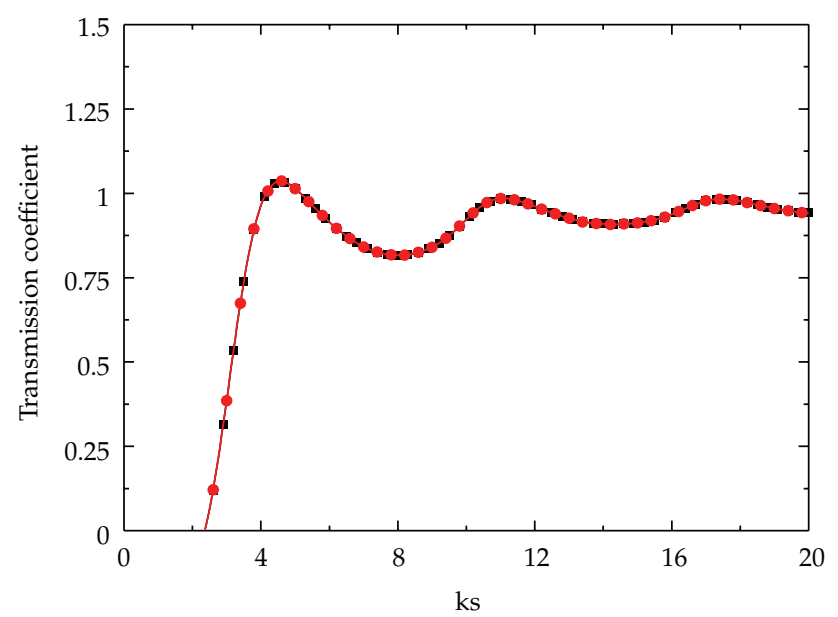

$\rightarrow$ PEMC cylinder copolarized component for $M \eta_{0}=\infty$

- PEC cylinder

(a)

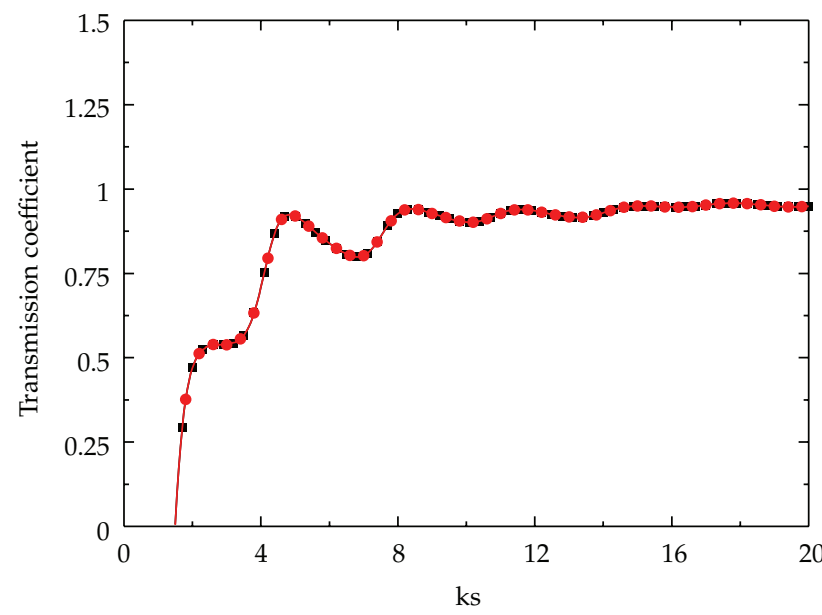

$\rightarrow$ PEMC cylinder copolarized component for $M \eta_{0}=\infty$

$\rightarrow$ PEC cylinder

(b)

Figure 2: (a) Slit transmission coefficient for $\theta_{0}=0, k d=0, k a=0.5$. (b) Slit transmission coefficient for $\theta_{0}=0, k d=5, k a=0.5$.

If the plane wave is restricted such that the incident field does not illuminate the lower faces of the half planes, the total field in the forward direction is given by [10]

$$
E^{t}=E^{i}+E^{s},
$$

where 


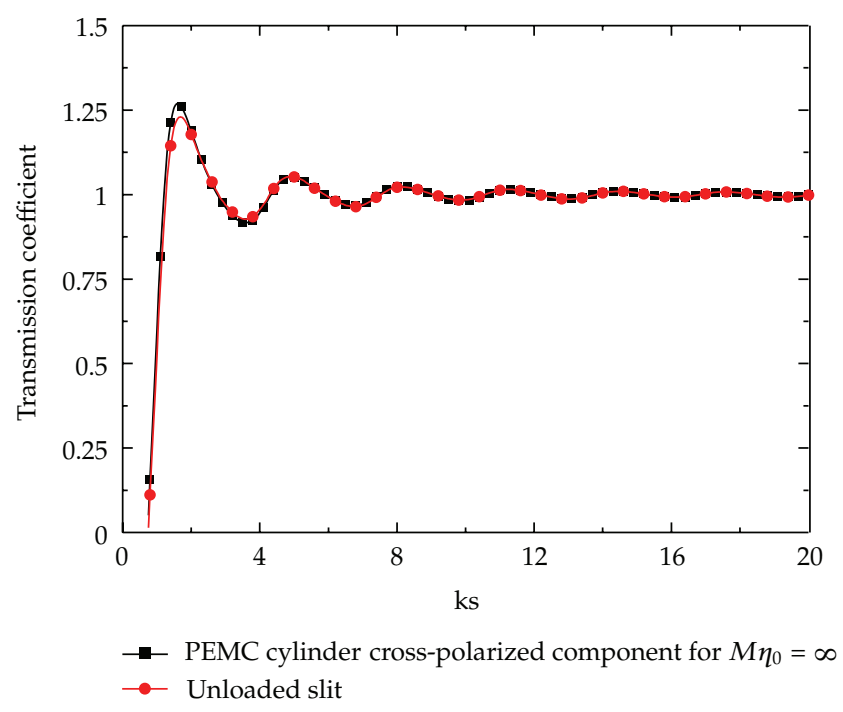

Figure 3: Slit transmission coefficient for $\theta_{0}=0, k d=0, k a=0$.

$$
\begin{gathered}
E^{s}=E^{s 1}+E^{s 2}+E^{s 3}, \\
E^{s 1}=\frac{\pi}{2 j} H_{0}\left(k \rho_{1}\right)\left[\exp \left(-j k s \sin \theta_{0}\right)\right] D\left(\phi_{1}, \phi_{01}, n_{1}\right)+c_{3} F\left(\phi_{1}, s_{1}, \phi_{31}, n_{1}\right)+c_{2} F\left(\phi_{1}, 2 s, \phi_{21}, n_{1}\right), \\
E^{s 2}=\frac{\pi}{2 j} H_{0}\left(k \rho_{2}\right)\left[\exp \left(+j k s \sin \theta_{0}\right)\right] D\left(\phi_{2}, \phi_{02}, n_{1}\right)+c_{3} F\left(\phi_{1}, s_{1}, \phi_{32}, n_{1}\right)+c_{2} F\left(\phi_{1}, 2 s, \phi_{12}, n_{2}\right), \\
E^{s 3}=\frac{\pi}{2 j} H_{0}\left(k \rho_{3}\right)\left[\exp \left(-j k d \cos \theta_{0}\right)\right] D\left(\phi_{3}, \phi_{03}, a\right)+c_{1} G\left(\phi_{3}, s_{1}, \phi_{13}, a\right)+c_{2} G\left(\phi_{3}, s_{2}, \phi_{23}, a\right),
\end{gathered}
$$

where $n_{1}=(2 \pi-\alpha) / \pi$ and $n_{2}=(2 \pi-\beta) / \pi$ and $c_{1}, c_{2}$ and $c_{3}$ are the unknown strengths of the line sources at wedge edges and along the cylinder axis, respectively. Let the incoming plane wave be incident from above the slit and the observation point be below. Further, let $\theta_{0}$ between the incoming plane wave and the normal to the plane of the screen (measured from the positive $y$-axis), and let $\theta$ represent the angle between the observation point and normal to the screen (measured from the negative $y$-axis). All angles are considered positive if measured counterclockwise with respect to the normal and negative if clockwise. When the observation point is far from the edges as compared to the width of double wedge $k \rho / 2 s \gg 1$, approximate relations between them can be simply stated. Therefore, wellknown far field conditions are used in which $\phi_{0}=\phi_{01}=\phi_{03}=\pi / 2+\theta_{0}, \phi_{02}=\pi / 2-\theta_{0}$, $\phi_{1}=\phi_{3} \simeq 3 \pi / 2+\theta, \phi_{2} \simeq 3 \pi / 2-\theta, \phi_{12}=\phi_{21} \simeq \pi, \phi_{13}=\psi \simeq \tan ^{-1}(d / s), \phi_{31}=$ $\phi_{32} \simeq \pi+\psi, \phi_{23} \simeq \pi-\psi, \rho_{1} \simeq \rho-s \sin \theta, \rho_{2} \simeq \rho+s \sin \theta, \rho_{3} \simeq \rho-d \cos \theta$, and $s_{1}$ and $s_{2}$ are the distances between the edges of the two wedges and the cylinder axis, respectively. 


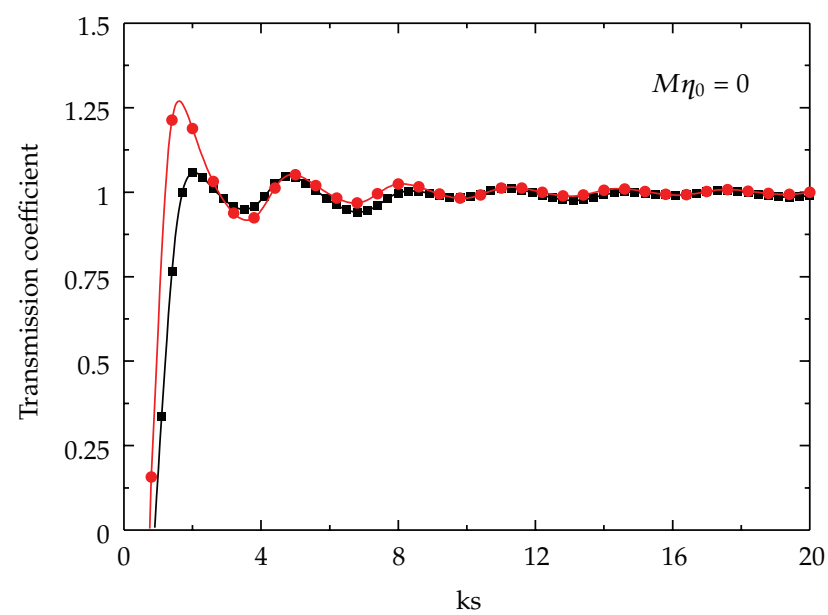

- PEMC cylinder copolarized component

$\rightarrow$ PEMC cylinder cross-polarized component

(a)

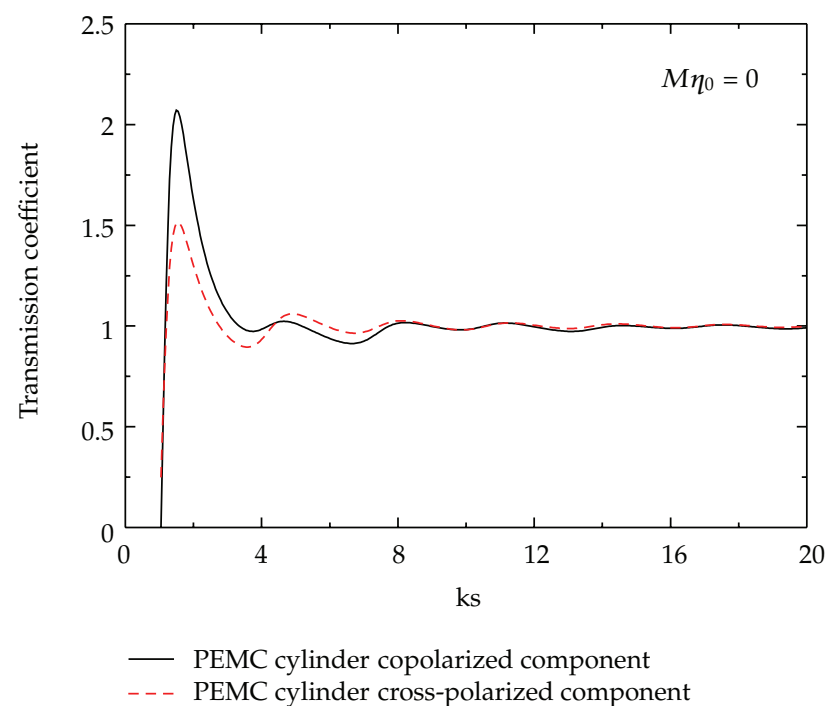

(b)

Figure 4: (a) Slit transmission coefficient for $\theta_{0}=0, k d=0, k a=0.5$. (b) Slit transmission coefficient for $\theta_{0}=0, k d=5, k a=0.5$.

To determine $c_{1}, c_{2}$ and $c_{3}$, the analysis of Karp and Russek [8] has been followed by imposing the requirement that the fields scattered by the two wedges and the cylinder be consistent with one another

$$
\begin{aligned}
2 c_{1}- & c_{2}\left[F\left(\phi_{31}, 2 s, \phi_{21}, n_{1}\right)+F\left(\phi_{21}, 2 s, \phi_{21}, n_{1}\right)\right]-c_{3}\left[F\left(\phi_{31}, s_{1}, \phi_{31}, n_{1}\right)+F\left(\phi_{21}, s_{1}, \phi_{31}, n_{1}\right)\right] \\
& =\exp \left(-j k s \sin \theta_{0}\right)\left[D\left(\phi_{31}, \phi_{01}, n_{1}\right)+D\left(\phi_{21}, \phi_{01}, n_{1}\right)\right], \\
2 c_{2}- & c_{1}\left[F\left(\phi_{32}, 2 s, \phi_{12}, n_{2}\right)+F\left(\phi_{12}, 2 s, \phi_{12}, n_{2}\right)\right]-c_{3}\left[F\left(\phi_{32}, s_{2}, \phi_{32}, n_{2}\right)+F\left(\phi_{12}, s_{2}, \phi_{32}, n_{2}\right)\right] \\
& =\exp \left(j k s \sin \theta_{0}\right)\left[D\left(\phi_{32}, \phi_{02}, n_{2}\right)+D\left(\phi_{12}, \phi_{02}, n_{2}\right)\right],
\end{aligned}
$$




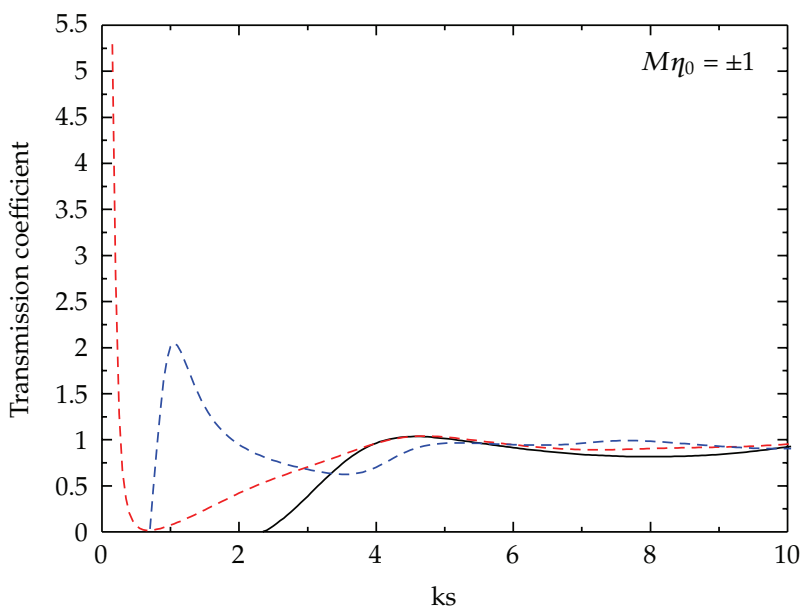

(a)

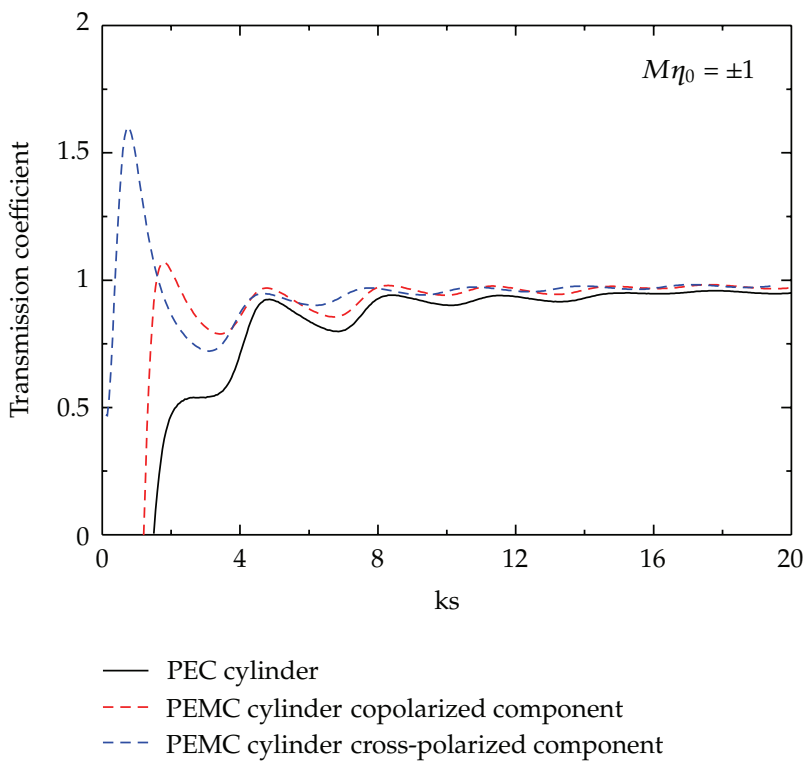

(b)

Figure 5: (a) Slit transmission coefficient for $\theta_{0}=0, k d=0, k a=0.5$. (b) Slit transmission coefficient for $\theta_{0}=0, k d=5, k a=0.5$.

$$
\begin{aligned}
2 c_{3}- & c_{1}\left[G\left(\phi_{13}, s_{1}, \phi_{13}, a\right)+G\left(\phi_{23}, s_{1}, \phi_{13}, a\right)\right]-c_{2}\left[G\left(\phi_{13}, s_{2}, \phi_{23}, a\right)+G\left(\phi_{23}, s_{2}, \phi_{23}, a\right)\right] \\
& =\exp \left(-j k d \cos \theta_{0}\right)\left[D\left(\phi_{13}, \phi_{03}, a\right)+D\left(\phi_{23}, \phi_{03}, a\right)\right] .
\end{aligned}
$$

By solving (4.3) for $c_{1}, c_{2}$, and $c_{3}$, the scattered field is found and is rewritten in the form 


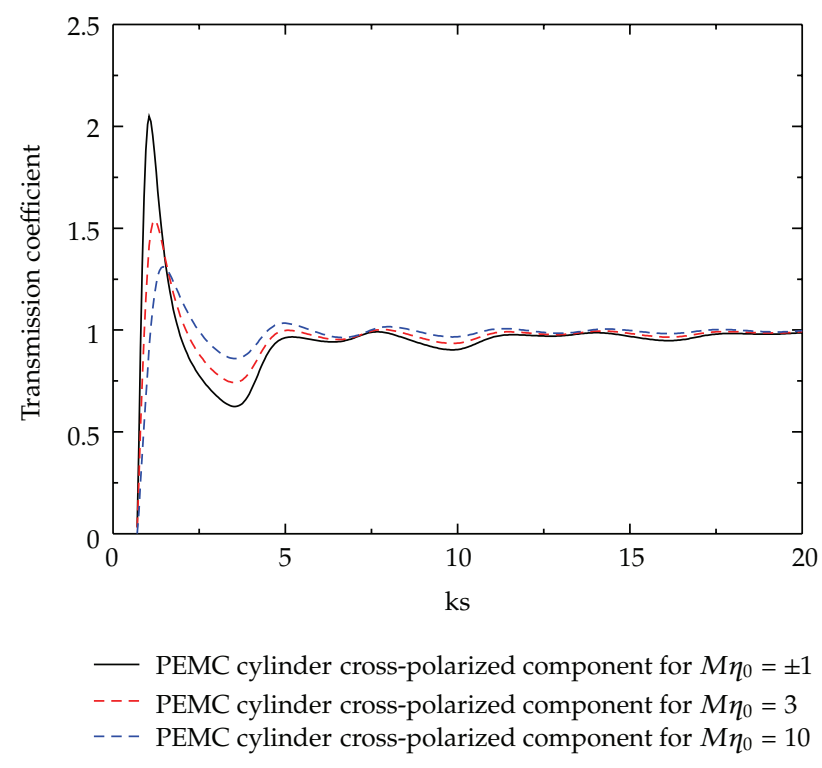

Figure 6: Slit transmission coefficient for $\theta_{0}=0, k d=0, k a=0.5$.

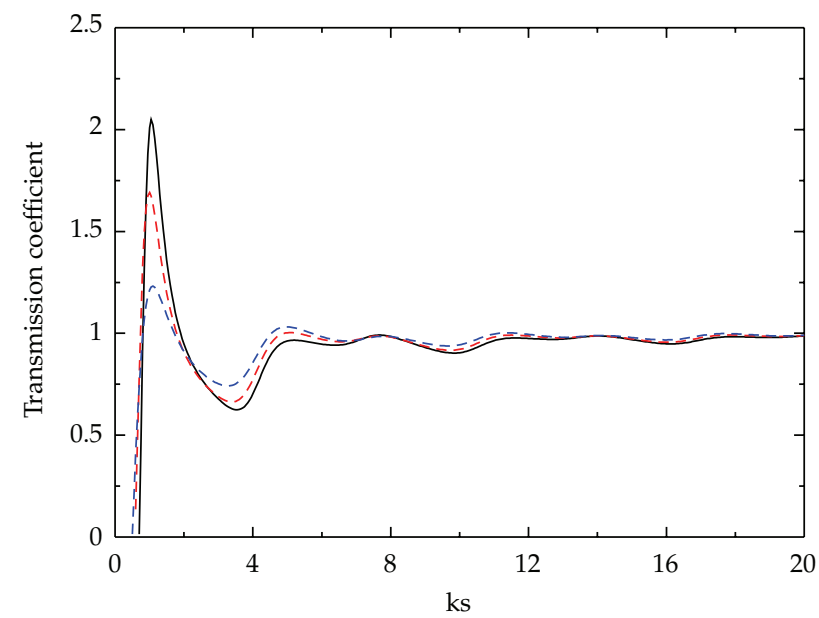

- PEMC cylinder-cross-polarized component for $k a=0.5$

- - PEMC cylinder-cross-polarized component for $k a=0.3$

- - PEMC cylinder-cross-polarized component for $k a=0.1$

Figure 7: Slit transmission coefficient for $\theta_{0}=0, k d=0, k a=0.5$.

$$
E^{S}=\frac{\exp (-j k \rho)}{\sqrt{\pi k \rho}} E\left(\theta, s, d, n_{1}, n_{2}, a\right),
$$

where the scattered field pattern $E\left(\theta, s, d, n_{1}, n_{2}, a\right)$ is obtained from (4.4). 


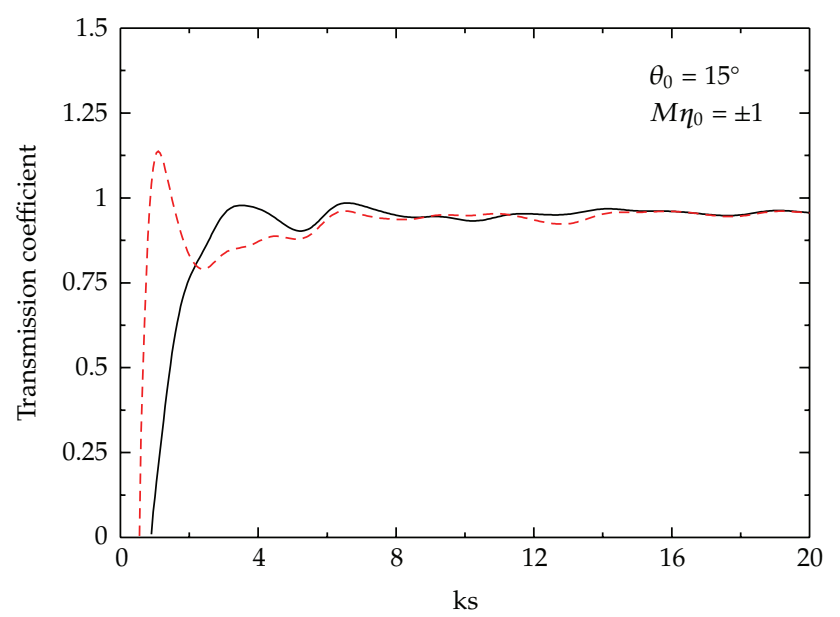

(a)

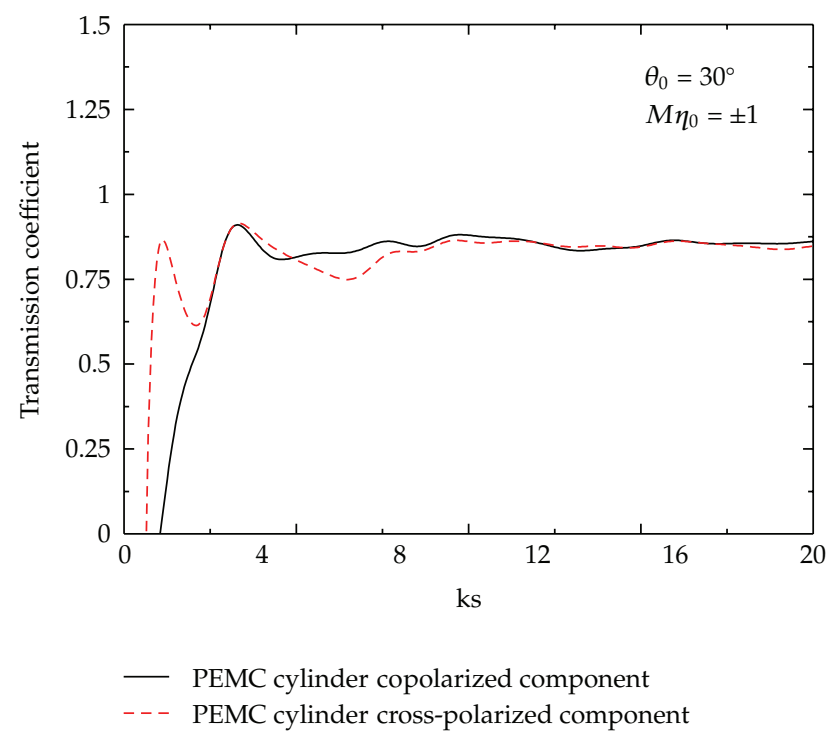

(b)

Figure 8: (a) Slit transmission coefficient for $k d=0, k a=0.1$. (b) Slit transmission coefficient for $k d=0$, $k a=0.1$.

The transmission coefficient $T$ for plane wave incidence is calculated using the expression given by Karp and Russek [8] as

$$
T=\frac{\operatorname{Re}[(1-j) \tilde{E}]}{2 k s},
$$

where $\widetilde{E}$ is $E\left(\theta, s, d, n_{1}, n_{2}, a\right)$ in the limit as $\theta$ approaches $\theta_{0}$. 


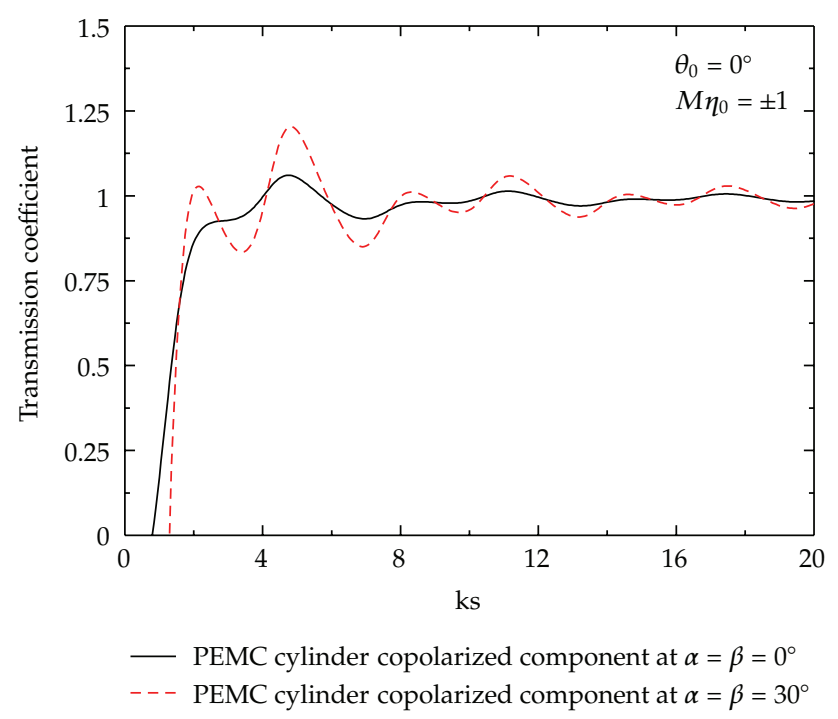

(a)

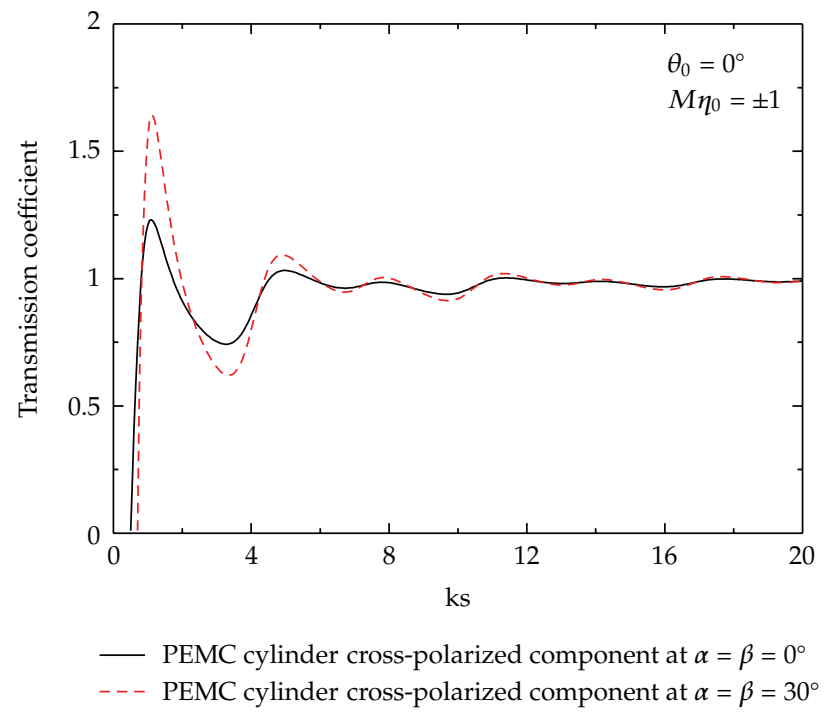

(b)

Figure 9: (a) Slit transmission coefficient for $k d=0, k a=0.1$. (b) Slit transmission coefficient for $k d=0$, $k a=0.1$.

\section{Results and Discussion}

Figure 1(a) shows the geometry which consists of PEC double wedge and a coated PEMC circular cylinder whereas Figure 1(b) contains an Uncoated PEMC cylinder placed under PEC wide double. In these figures, $d$ represents the distance of the PEMC cylinder from the edge of PEC wedge. In the first part of discussion, a comparison of transmission coefficients $\left(T_{c}\right)$ for PEC double wedge with zero wedge angle, loaded with PEC cylinder is made with 


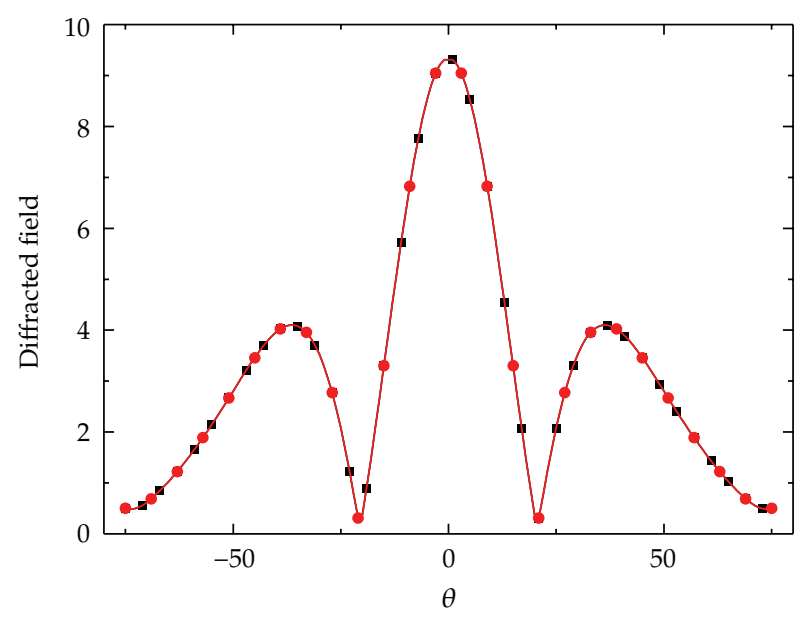

$\rightarrow$ PEMC cylinder copolarized component for $M \eta_{0}=\infty$

$\rightarrow$ PEC cylinder

(a)

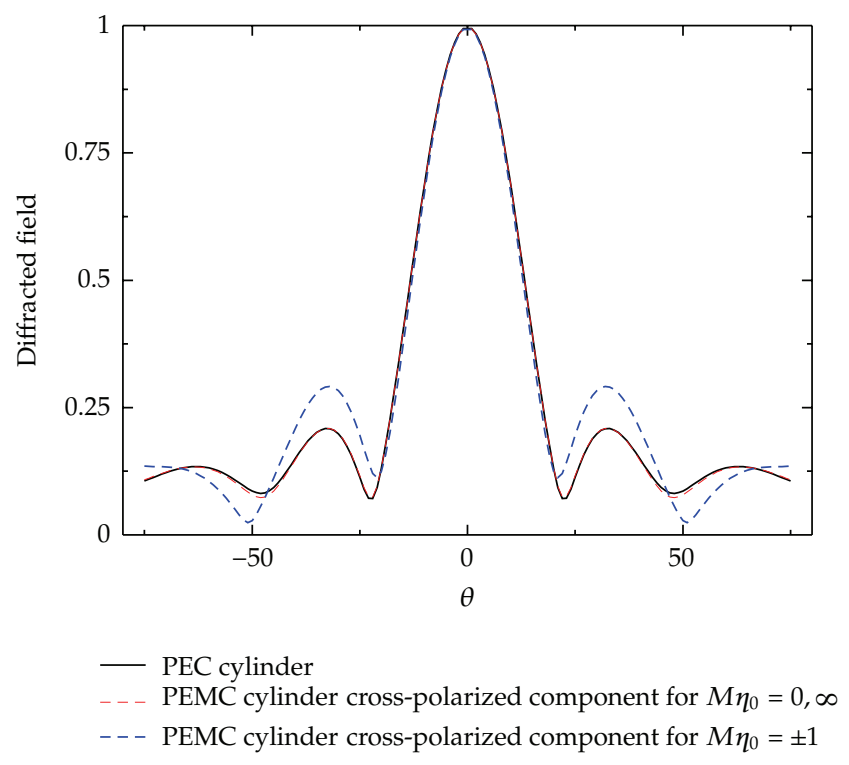

(b)

Figure 10: (a) Slit diffraction pattern for $\theta_{0}=0^{0}, k d=0, k a=0.5, k s=8$. (b) Slit diffraction pattern for $\theta_{0}=0^{0}, k d=0, k a=0.5, k s=8$.

the transmission coefficient for the slit when a uncoated PEMC cylinder is placed under the slit. Comparison of $T_{c}$ with both the copolarized $\left(T_{\mathrm{co}}\right)$ and cross-polarized $\left(T_{\text {cross }}\right)$ components of uncoated PEMC cylinder is studied. In all the cases cylinder radius $(k a)$ is taken as 0.5. Figures 2(a) and 2(b) show the comparison of $T_{c}$ with $T_{\mathrm{co}}$ when the cylinder is located at $k d=0$ and $k d=5$ from the slit, respectively. It can be observed that $T_{\mathrm{co}}$, in both the cases, 


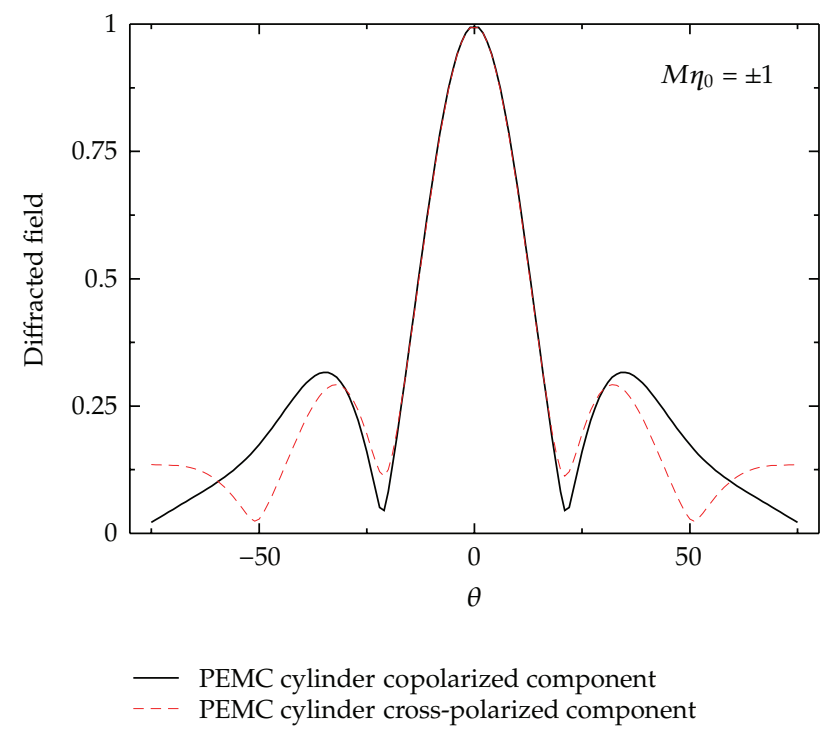

Figure 11: Slit diffraction pattern for $\theta_{0}=0^{0}, k d=0, k a=0.5, k s=8$.

show exactly the same behavior as that of $T_{c}$ when $M \eta_{0}=\infty$. These results are in fairly good agreement with the Elsherbeni's results [10]. Therefore, it is quite obvious that the uncoated PEMC cylinder behaves like PEC cylinder at $M \eta_{0}=\infty$. In Figure 3, a comparison of $T_{\text {cross }}$ has been made with the transmission coefficient $(T)$ for the slit when the cylinder radius $k a=0$, that is, an unloaded slit. It can be observed that the two coefficients have the same behavior. It is because the cross-polarized component of PEMC cylinder is zero at $M \eta_{0}=0$. In Figures 4 and 5, a comparison of $T_{\text {co }}$ and $T_{\text {cross }}$ for $M \eta_{0}=0$ and $M \eta_{0}= \pm 1$ at $k d=0$ and $k d=5$ are presented, respectively. In Figure $4(\mathrm{a})$, it can be seen that when the cylinder, with $M \eta_{0}=0$, is at $k d=0, T_{\text {co }}$ is less than that of $T_{\text {cross }}$, but when it is shifted below the center of the aperture plane, say at $k d=5, T_{\mathrm{co}}$ becomes larger than $T_{\text {cross }}$ which is obvious from Figure $4(\mathrm{~b})$. Moreover, the transmission coefficients oscillate with decreasing amplitude as expected and tend to unity as the slit width $k s$ tends to infinity. But, contrary to this effect, $T_{\text {cross }}$ remains larger at $k d=5$ when $M \eta_{0}= \pm 1$, as shown in Figure 5(b), whereas at $k d=0 T_{\text {cross }}$ is less than $T_{\text {co }}$ as shown in Figure 5(a). Moreover, it can be seen that when $k d=0, T_{\text {co }}$ shows almost similar behavior as that of $T_{c}$ but $T_{\text {cross }}$ is larger than $T_{c}$. When the cylinder is shifted to $k d=5$, both $T_{\text {(co) }}$ and $T_{\text {(cross) }}$ are larger than $T_{c}$. To further highlight the effect of $M \eta_{0}$ on $T_{\text {cross, }}$, Figure 6 shows that $T_{\text {cross }}$ is maximum when $M \eta_{0}= \pm 1$ and decreases for other values of $M \eta_{0}$. Similarly Figure 7 shows the effect of variation of $k a$ on $T_{\text {cross }}$ at $M \eta_{0}= \pm 1$. Obviously the value of $T_{\text {cross }}$ is larger for $k a=0.5$ and decreases for smaller values of $k a$. Both these figures are for $k d=0$. The behavior of $T_{\text {co }}$ and $T_{\text {cross }}$ for obliquely incident plane wave at $\theta_{0}=15^{0}$ and $\theta_{0}=30^{0}$ for $k a=0.1, k d=0$ and $M \eta_{0}= \pm 1$ is shown in Figures 8(a) and $8(\mathrm{~b})$. At $\theta_{0}=15^{0}, T_{\text {cross }}$ is higher than unity in the lower range of $k s(k s \leq 3)$ and is larger than $T_{\mathrm{co}}$. For the same cylinder parameters but with $\theta_{0}=30^{\circ}$, both $T_{\mathrm{co}}$ and $T_{\text {cross }}$ becomes less than unity. However, $T_{\text {cross }}$ oscillates with greater amplitude as compared to $T_{\mathrm{co}}$. Hence incident angle effects the peak locations of $T_{\text {co }}$ and $T_{\text {cross. }}$. To see the effect of interior wedge angle on the transmission coefficients for $M \eta_{0}= \pm 1, k a=0.1$, it is observed that as the wedge angle is increased, the amplitude of oscillation in both $T_{\mathrm{co}}$ and $T_{\text {cross }}$ is increased, that is, the interior wedge angle effects the levels of maxima and minima of the oscillation in both the 


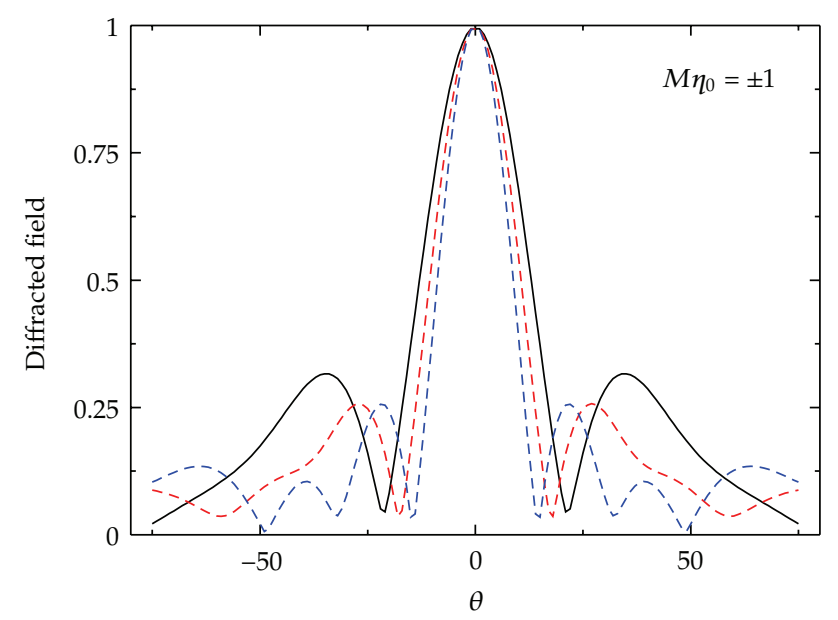

- PEMC cylinder-co-polarized component for $k s=8$
-- PEMC cylinder-co-polarized component for $k s=10$
-- PEMC cylinder-co-polarized component for $k s=12$

(a)

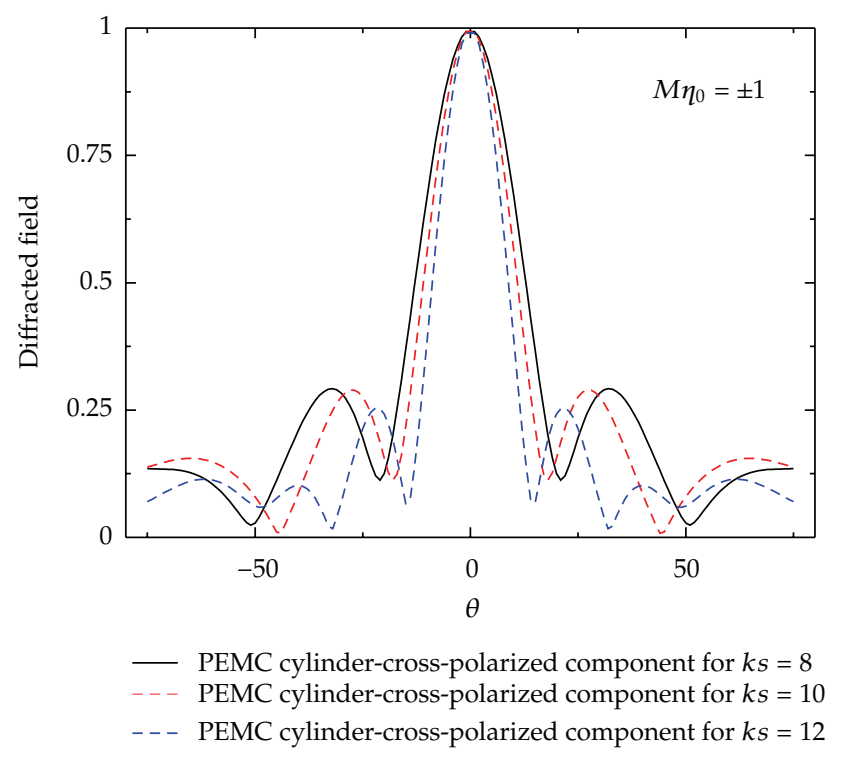

(b)

Figure 12: (a) Slit diffraction pattern for $\theta_{0}=0^{0}, k d=0, k a=0.5$. (b) Slit diffraction pattern for $\theta_{0}=0^{0}$, $k d=0, k a=0.5$.

cases, however this effect is more dominant in $T_{\text {cross }}$ as compared to $T_{\text {co }}$ as shown in Figures $9(\mathrm{a})$ and $9(\mathrm{~b})$.

In the second part of discussion, normalized diffraction pattern of the slit loaded with PEC cylinder $\left(D_{c}\right)$ compared with the corresponding normalized diffraction patterns in the presence of uncoated PEMC cylinder is presented. Comparison between copolarized 


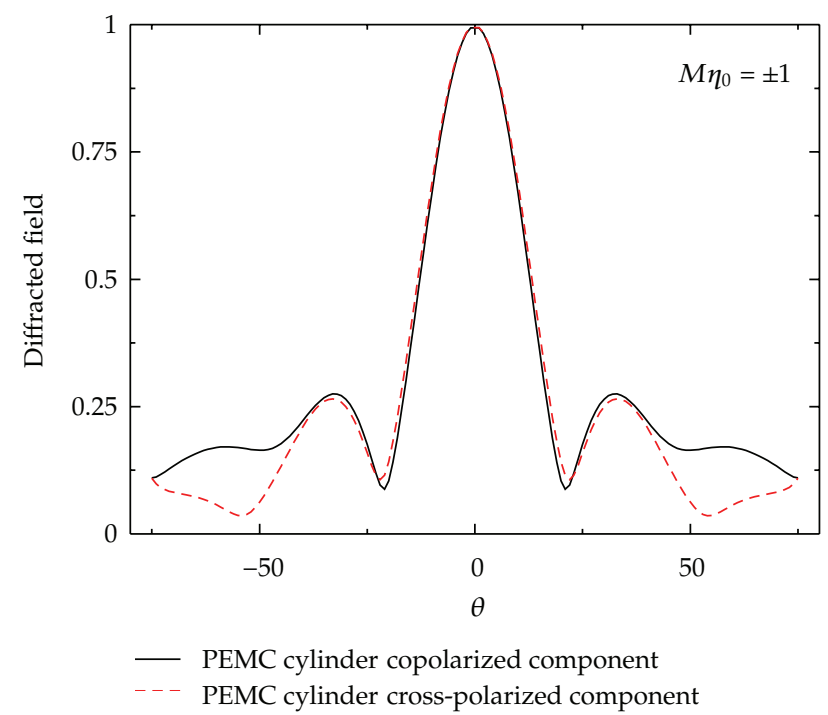

Figure 13: Slit diffraction pattern for $\theta_{0}=0^{0}, k d=1.5, k a=0.5, k s=8$.

$\left(D_{(\text {co })}\right)$ and cross-polarized $\left(D_{\text {(cross) }}\right)$ components of PEMC cylinder for different values of $M \eta_{0}$ is made. In all the cases cylinder radius $k a$ is taken as 0.5 . Figure 10 (a) presents $D_{c}$ compared with $D_{(\text {co })}$ for $k d=0$ and $k s=8$. The solid curve in the figure represents $D_{c}$. It is observed that $D_{\text {(co) }}$ shows similar behavior as that of $D_{c}$ for $M \eta_{0}=\infty$. Moreover, in Figure 10(b) it can be observed that $D_{\text {(cross) }}$ for both $M \eta_{0}=0$ and $M \eta_{0}=\infty$ gives the same diffraction patterns as that of an unloaded slit $(D)$ which is in good agreement with the published work [6]. This shows that cross-polarized component exists only for $M \eta_{0}= \pm 1$ and becomes zero for other values of $M \eta_{0}$. To further investigate the effect of $M \eta_{0}$ on $D_{\text {(co) }}$ and $D_{\text {(cross) }}$, Figure 11 shows the comparison of both these diffraction patterns for $M \eta_{0}= \pm 1$. It can be seen that the beam width for cross-polarized component is less than the beam width of copolarized component. To see the effect of slit width on $D_{(\text {co })}$ and $D_{\text {(cross) }}$, the plots for different values of $k s$ with $k d=0$ and $M \eta_{0}= \pm 1$ are shown in Figures 12(a) and 12(b). It is observed that the number of side lobes increases with the increase in slit width for both $D_{(\text {co) }}$ and $D_{\text {(cross) }}$. When the cylinder is shifted to $k d=1.5$ for $k s=8$ and $M \eta_{0}= \pm 1$, comparison of $D_{(\text {co })}$ and $D_{\text {(cross) }}$ shown in Figure 13, reflects almost the similar behavior.

In the third part of discussion, the transmission coefficient of coated PEMC cylinder is presented. Behavior of both copolarized $\left(T_{(\mathrm{co})}^{c}\right)$ and cross-polarized $\left(T_{(\mathrm{cross})}^{c}\right)$ components of coated PEMC cylinder is discussed. In all the plots radius of Uncoated cylinder is taken as $a=0.2 \mathrm{~cm}$ and that of coated cylinder as $b=0.3 \mathrm{~cm}$. The validity of the code has been checked by making the coating equal to zero. Results are found to be in agreement with Uncoated PEMC cylinder. Comparison of $T_{\text {co }}^{c}$ and $T_{\text {cross }}^{c}$ for $M \eta_{1}= \pm 1$ at $k d=0$ and $k d=5$ with relative permitivity $\epsilon_{r}=-1.5$ and relative permeability $\mu_{r}=-1$, are shown in Figures 14(a) and 14(b), respectively. It can be seen that in both the cases, $T_{\mathrm{co}}^{c}$ is larger than $T_{\text {cross }}^{c}$, which is contrary to Uncoated PEMC cylinder in which $T_{\text {co }}$ remains less than $T_{\text {cross }}$ for $M \eta_{0}= \pm 1$. Furthermore, it is observed that the transmission coefficient is large in the presence of coated PEMC cylinder as compared to PC cylinder. In both the cases $T_{\text {co }}^{c}$ and $T_{\text {cross }}^{c}$ are greater than unity whereas $T_{c}$, in general, remains less than unity. The variation in the radius of coated cylinder also effects 


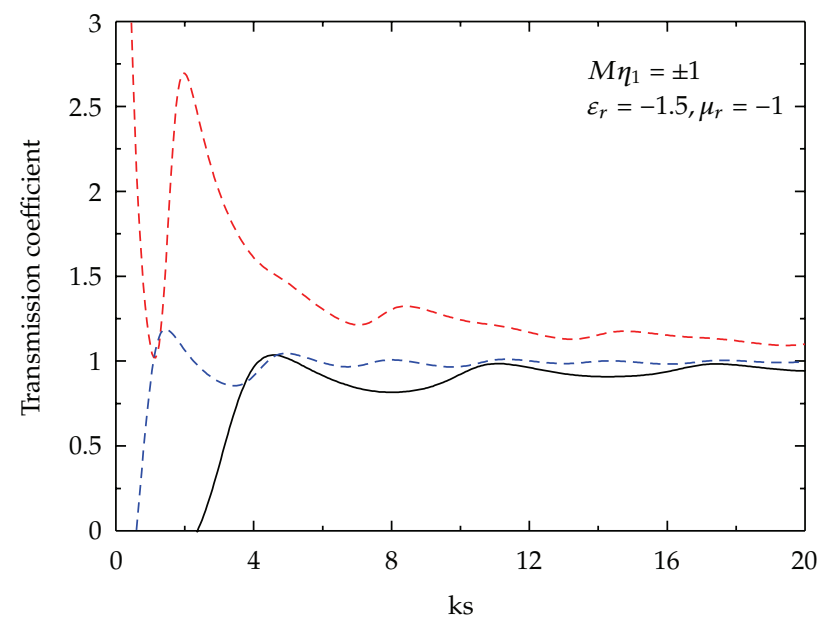

(a)

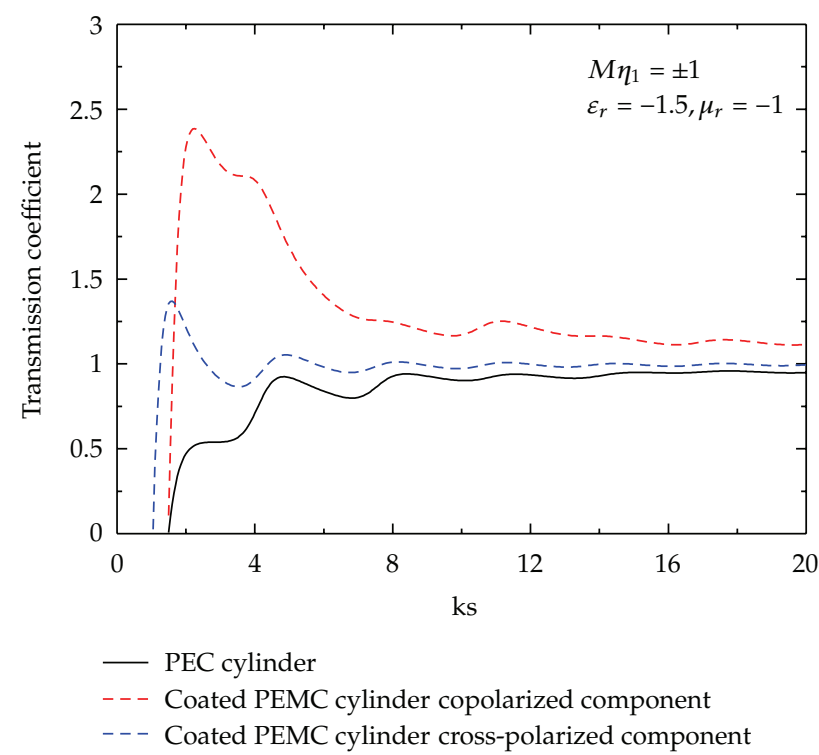

(b)

Figure 14: (a) Slit diffraction pattern for $\theta_{0}=0^{0}, k d=0, a=0.2 \mathrm{~cm}, b=0.3 \mathrm{~cm}$. (b) Slit diffraction pattern for $\theta_{0}=0^{0}, k d=5, a=0.2 \mathrm{~cm}, b=0.3 \mathrm{~cm}$.

the behavior of $T_{\text {co }}^{c}$ and $T_{\text {cross }}^{c}$ as shown in Figure 15. Figure 15(b) shows that $T_{\text {co }}^{c}$ oscillates with greater amplitude as the the value of $b$ is increased. However, $T_{\text {(cross) }}^{c}$ does not show considerable change in behavior with the increase in radius $b$ as hi-lighted in Figure 15(a). The behavior of $T_{\mathrm{co}}^{c}$ and $T_{\text {cross }}^{c}$ for oblique incidence is shown in Figure 16 for incident angles $\theta_{0}=20^{\circ}$ and $\theta_{0}=30^{\circ}$. Figure 16 (a) shows that $T_{\text {cross }}^{c}$ gets less than unity as the angle of incidence is increased from zero, whereas the amplitude of oscillation for $T_{\mathrm{co}}^{c}$ decreases with the increase of incidence angle $\theta_{0}$ as shown in Figure 16(b). All the plots of Figures 15 and 16 


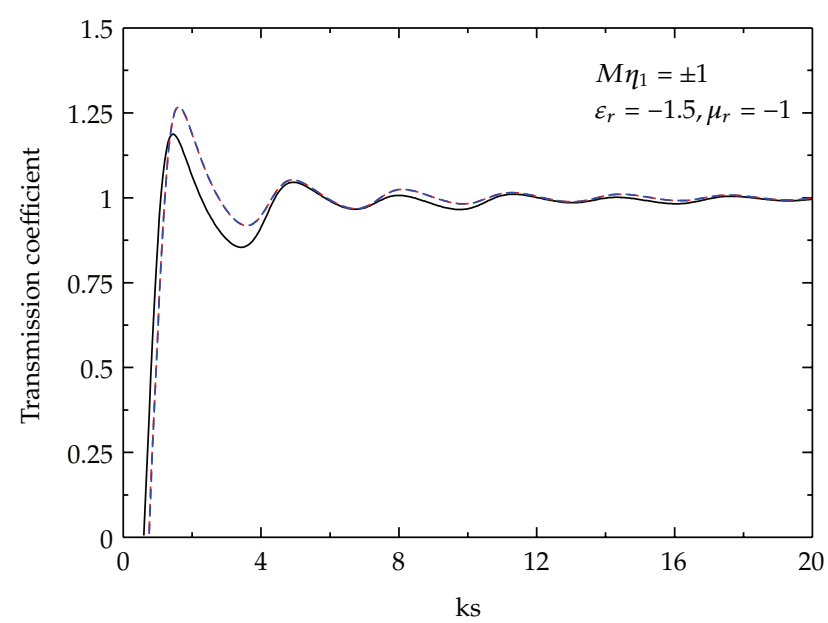

- Coated PEMC cylinder cross-polarized $(b=0.3 \mathrm{~cm})$

- - Coated PEMC cylinder cross-polarized $(b=0.4 \mathrm{~cm})$

-- - Coated PEMC cylinder cross-polarized $(b=0.6 \mathrm{~cm})$

(a)

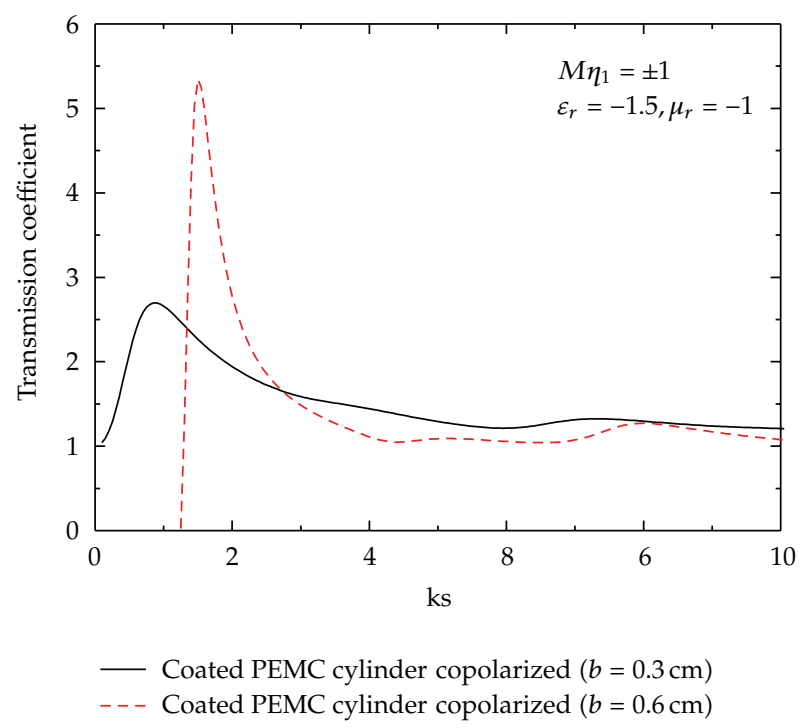

(b)

Figure 15: (a) Slit transmission coefficient for $\theta_{0}=0, k d=0$. (b) Slit transmission coefficient for $\theta_{0}=0$, $k d=0$.

are for for $M \eta_{1}= \pm 1$. Further, it can be seen in Figure 17 that interior wedge angle effects the peak-to-peak values of the oscillations both in the case of $T_{\mathrm{co}}^{c}$ and $T_{\text {cross }}^{c}$. In case of $T_{\text {cross }}^{c}$ as shown in Figure 17(a), the oscillations are always around unity and decreases with increasing $k s$ whereas in case of $T_{\mathrm{co}}^{c}$ as shown in Figure 17(b), the oscillations are larger and are greater than unity. The plots for DPS-coated cylinder show almost similar behavior as that of DNG coated cylinder. 


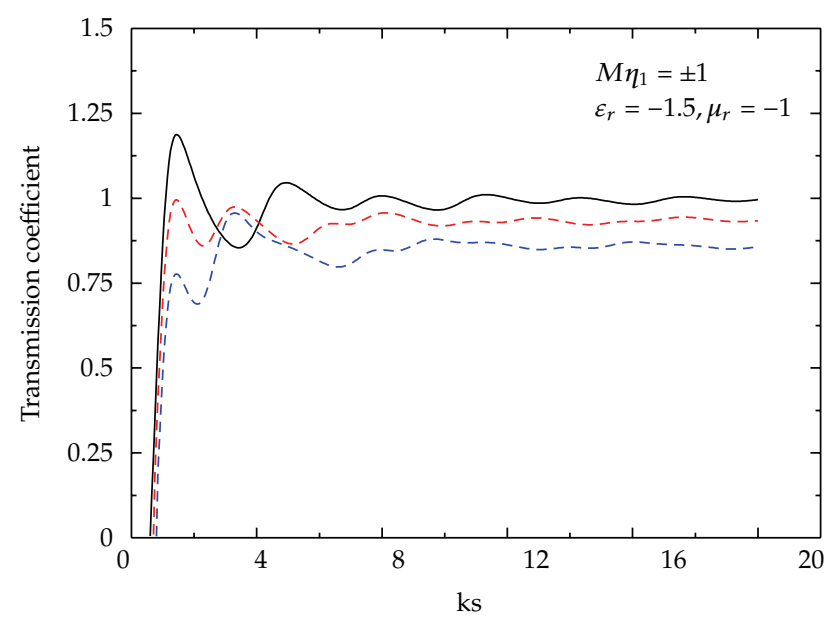

- Coated PEMC cylinder cross-polarized $\left(\theta_{0}=0^{\circ}\right)$
--- Coated PEMC cylinder cross-polarized $\left(\theta_{0}=20^{\circ}\right)$
--- Coated PEMC cylinder cross-polarized $\left(\theta_{0}=30^{\circ}\right)$

(a)

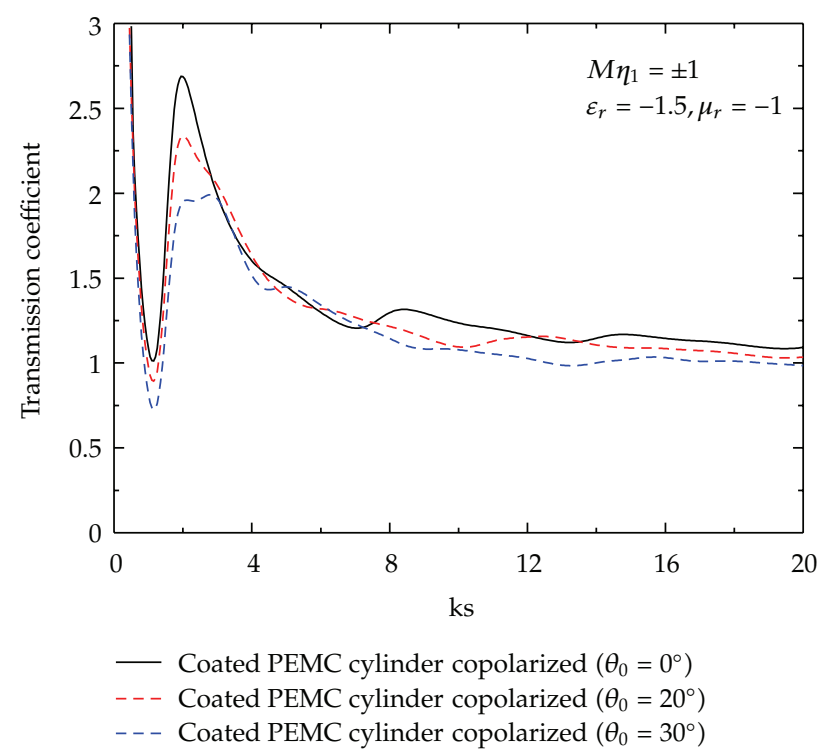

(b)

Figure 16: (a) Slit transmission coefficient for $\theta_{0}=0, k d=0, a=0.2 \mathrm{~cm}, b=0.3 \mathrm{~cm}$. (b) Slit transmission coefficient for $\theta_{0}=0, k d=0, a=0.2 \mathrm{~cm}, b=0.3 \mathrm{~cm}$.

In the last part of discussion, diffraction pattern of wide double wedge in the presence of coated PEMC cylinder is presented. In Figure 18, the effect of $M \eta_{1}$ on the diffraction pattern is shown. Behavior of both copolarised $\left(D_{(\mathrm{co})}^{c}\right)$ and cross-polarized $\left(D_{\text {(cross) }}^{c}\right)$ components of coated PEMC cylinder with $\epsilon_{r}=1.5$ and $\mu_{r}=1$ for $k d=0$ and $k s=8$ is studied. In both the cases, it can be seen that $D_{(\mathrm{co})}^{c}$ and $D_{\text {(cross) }}^{c}$ show slight different 


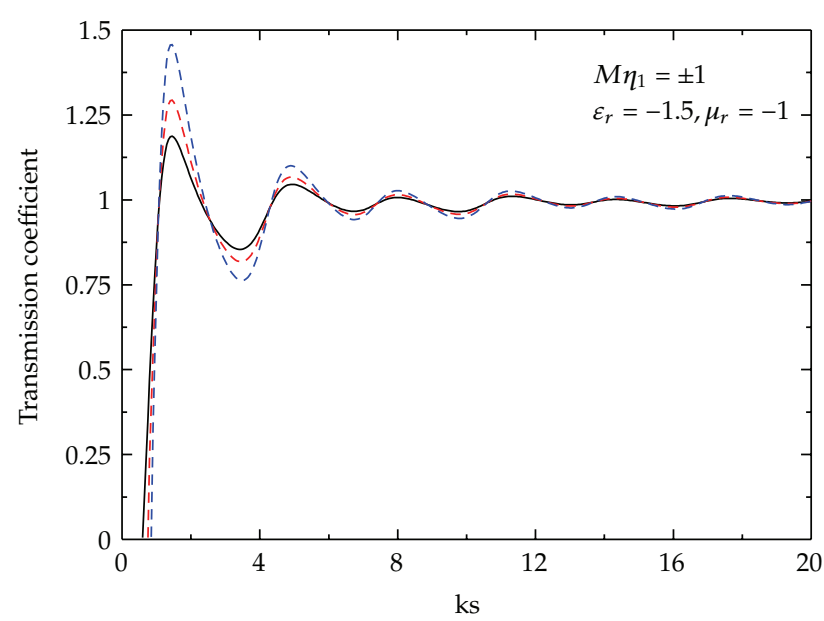

Coated PEMC cylinder cross-polarized $\left(\alpha=\beta=0^{\circ}\right)$

- - - Coated PEMC cylinder cross-polarized $\left(\alpha=\beta=20^{\circ}\right)$

- - - Coated PEMC cylinder cross-polarized $\left(\alpha=\beta=30^{\circ}\right)$

(a)

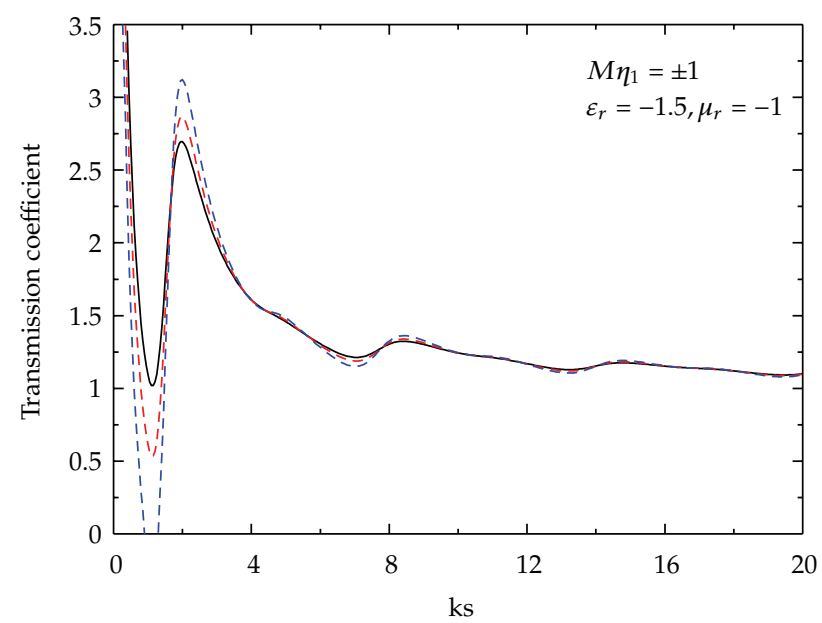

- Coated PEMC cylinder copolarized $\left(\alpha=\beta=0^{\circ}\right)$

- - Coated PEMC cylinder copolarized $\left(\alpha=\beta=20^{\circ}\right)$

- - Coated PEMC cylinder copolarized $\left(\alpha=\beta=30^{\circ}\right)$

(b)

Figure 17: (a) Slit transmission coefficient for $\theta_{0}=0, k d=0, a=0.2 \mathrm{~cm}, b=0.3 \mathrm{~cm}$. (b) Slit transmission coefficient for $\theta_{0}=0, k d=0, a=0.2 \mathrm{~cm}, b=0.3 \mathrm{~cm}$.

behavior for $M \eta_{1}=1$ as compared to other values of $M \eta_{1}$. Figure 19 shows the variation in $D_{\text {(co) }}^{c}$ and $D_{\text {(cross) }}^{c}$ for $\epsilon_{r}=1.5, \mu_{r}=1$ and $k d=0$ with respect to the slit width. It is observed that both $D_{(\mathrm{co})}^{c}$ and $D_{(\mathrm{cross})}^{c}$ show different behavior for different values of slit widths. 


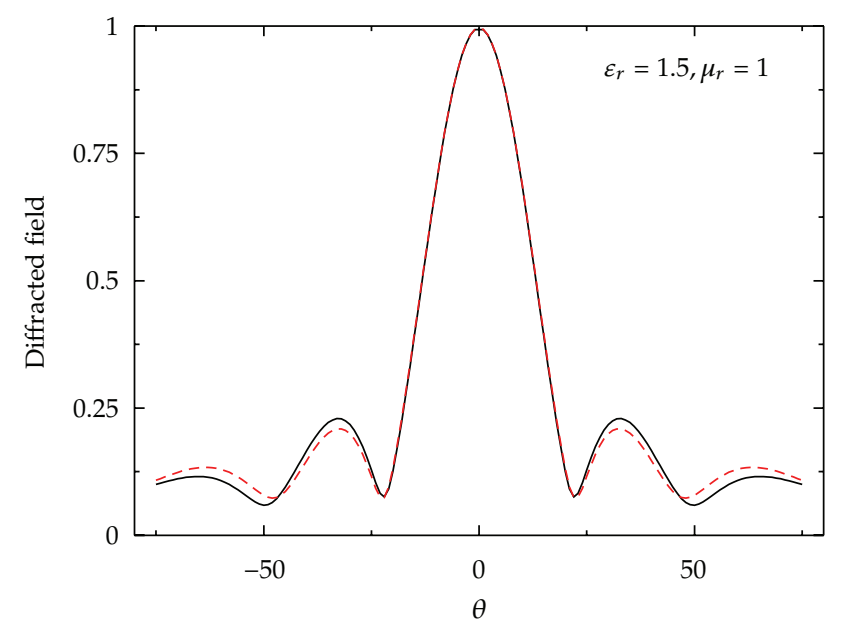

- Coated PEMC cylinder cross-polarized component with $M \eta_{1}= \pm 1$

- - Coated PEMC cylinder cross-polarized component with $M \eta_{1}=0, \infty$

(a)

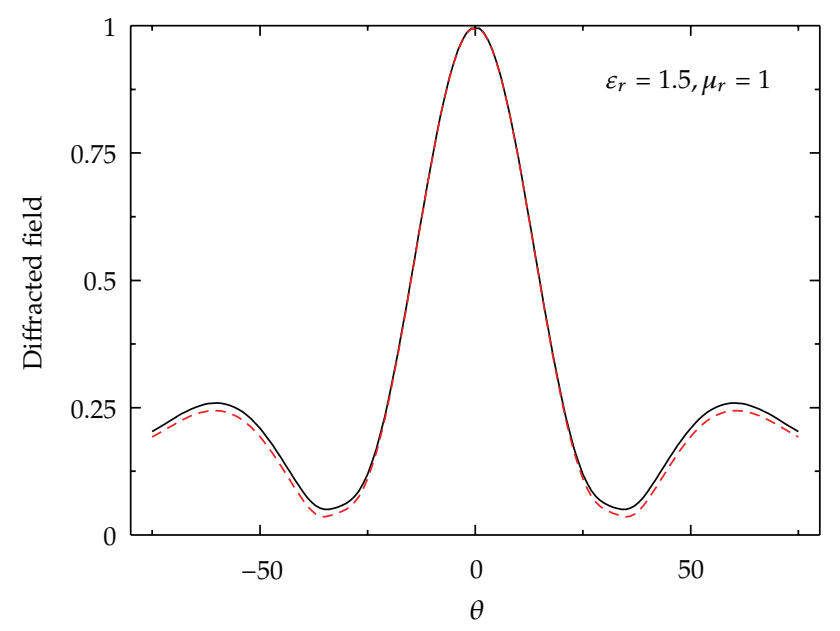

— Coated PEMC cylinder copolarized component with $M \eta_{1}= \pm 1$

- - Coated PEMC cylinder copolarized component with $M \eta_{1}=0, \infty$

(b)

Figure 18: (a) Slit diffraction pattern for $\theta_{0}=0^{0}, k s=8, k d=0, a=0.2, b=0.3$. (b) Slit diffraction pattern for $\theta_{0}=0^{0}, k s=8, k d=0, a=0.2, b=0.3$.

\section{Conclusion}

The transmission coefficient and the diffraction pattern of three scatterers, that is, two PEC parallel wedges in the presence of a coated PEMC cylinder are presented. The results show that the transmission coefficient has a high value in the presence of a PEMC cylinder as compared to PEC cylinder. Furthermore, it is observed that the transmission coefficient varies under particular conditions such as by either shifting the cylinder below the center of the 


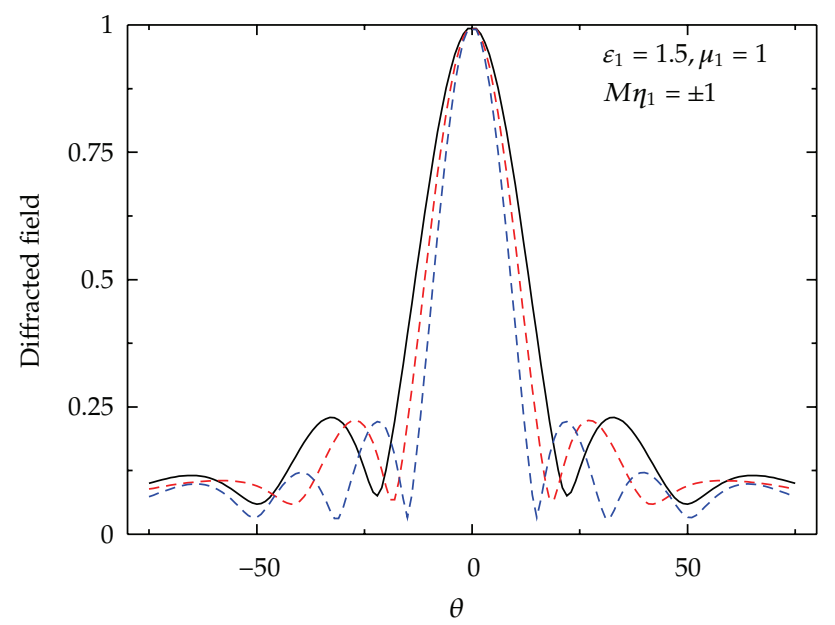

- Coated PEMC cylinder cross-polarized component for $k s=8$

- - - Coated PEMC cylinder cross-polarized component for $k s=10$

- - - Coated PEMC cylinder cross-polarized component for $k s=12$

(a)

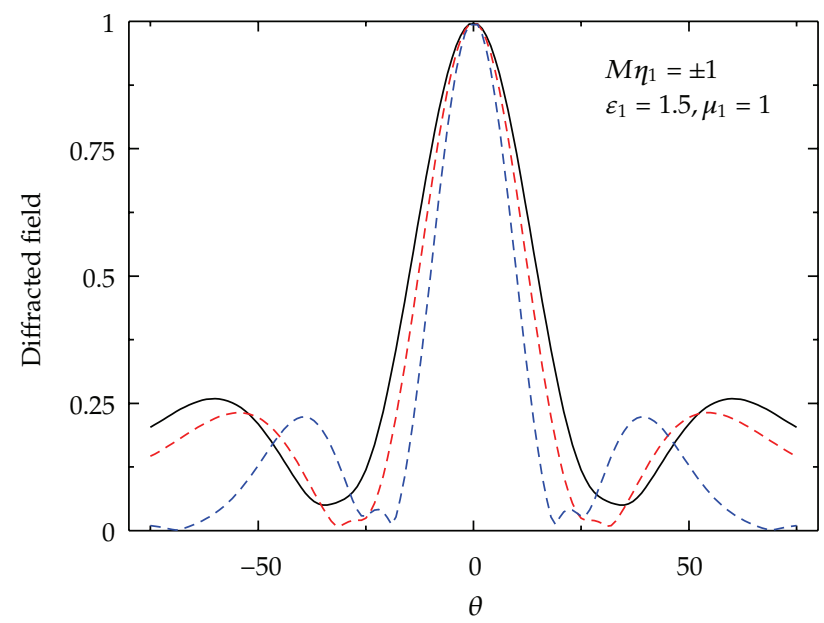

- Coated PEMC cylinder cross-polarized component for $k s=8$
-- Coated PEMC cylinder cross-polarized component for $k s=10$
-- Coated PEMC cylinder cross-polarized component for $k s=12$

(b)

Figure 19: (a) Slit diffraction pattern for $\theta_{0}=0^{0}, k d=0, a=0.2, b=0.3$. (b) Slit diffraction pattern for $\theta_{0}=0^{0}, k d=0, a=0.2, b=0.3$.

aperture plane of double wedge or by coating the PEMC cylinder with DPS or DNG materials. Variations in the transmission coefficient with respect to different values of admittance parameter for Uncoated and coated PEMC cylinder is also studied. It is found that the behavior of $T_{(\mathrm{co})}$ and $T_{\text {(cross) }}$ of an Uncoated PEMC cylinder and $T_{(\mathrm{co})}^{c}$ and $T_{\text {(cross) }}^{c}$ of coated PEMC cylinder not only varies with the incident angles of the original plane wave but also show cosiderable change in the behavior by changing the interior wedge angles. 


\section{References}

[1] A. Z. Elsherbeni and M. Hamid, "Diffraction by a wide double wedge with rounded edges," IEEE Transactions on Antennas and Propagation, vol. 33, no. 9, pp. 1012-1015, 1985.

[2] V. Twersky, "Multiple scattering of radiation by an arbitrary planar configuration of parallel cylinders and by two parallel cylinders," Journal of Applied Physics, vol. 23, pp. 407-414, 1952.

[3] S. Karp and J. Radlow, "On resonance in infinite grating of cylinders," IRE Transactions on Antennas and Propagation, vol. 4, no. 4, p. 654, 1956.

[4] Norman Zitron and Samuel N. Karp, "Higher-order approximations in multiple scattering. I. Twodimensional scalar case," Journal of Mathematical Physics, vol. 2, pp. 394-402, 1961.

[5] K. Hongo and G. Ishii, "Diffraction of electromagnetic plane wave by thick slit," IERE Transactions on Antennas and Propagation, vol. 26, no. 3, pp. 494-499, 1978.

[6] M. Naveed and Q. A. Naqvi, "Scattering of electromagnetic plane waves by a perfectly conducting slit and a PEMC parallel cylinder," Progress in Electromagnetic Reasearch, vol. 1, pp. 45-58, 2008.

[7] P. C. Clemmow, "Edge currents in diffraction theory," IRE Transactions on Antennas and Propagation, vol. 4, pp. 282-287, 1956.

[8] S. N. Karp and A. Russek, “Diffraction by a wide slit," Journal of Applied Physics, vol. 27, pp. 886-894, 1956.

[9] A. Z. Elsherbeni and M. Hamid, "Diffraction by a wide double wedge," IEEE Transactions on Antennas and Propagation, vol. 32, no. 11, pp. 1262-1265, 1984.

[10] A. Z. Elsherbeni and M. Hamid, "Scattering by a double wedge and a parallel cylinder," International Journal of Electronics, vol. 60, no. 3, pp. 367-380, 1986.

[11] I. V. Lindell and A. H. Sihvola, "Perfect electromagnetic conductor," Journal of Electromagnetic Waves and Applications, vol. 19, no. 7, pp. 861-869, 2005.

[12] I. V. Lindell and A. H. Sihvola, "Realization of the PEMC boundary," IEEE Transactions on Antennas and Propagation, vol. 53, no. 9, pp. 3012-3018, 2005.

[13] I. V. Lindell and A. H. Sihvola, "Transformation method for problems involving perfect electromagnetic conductor (PEMC) structures," IEEE Transactions on Antennas and Propagation, vol. 53, no. 9, pp. 3005-3011, 2005.

[14] R. Ruppin, "Scattering of electromagnetic radiation by a perfect electromagnetic conductor cylinder," Journal of Electromagnetic Waves and Applications, vol. 20, no. 13, pp. 1853-1860, 2006.

[15] M. A. Fiaz, A. Aziz, A. Ghaffar, and Q. A. Naqvi, "High frequency expression for the filed in the caustic region of a PEMC gregorian system using Maslov's method," Progress In Electromagnetics Research, vol. 81, pp. 135-148, 2008.

[16] M. A. Fiaz, A. Ghaffar, and Q. A. Naqvi, "High-frequency expressions for the field in the caustic region of a PEMC cylindrical reflector using Maslov's method," Journal of Electromagnetic Waves and Applications, vol. 22, no. 2-3, pp. 385-397, 2008.

[17] S. Ahmed and Q. A. Naqvi, "Electromagnetic scattering from a perfect electromagnetic conductor cylinder buried in a dielectric half-space," Progress in Electromagnetics Research, vol. 78, pp. 25-38, 2008.

[18] S. Ahmed and Q. A. Naqvi, "Electromagnetic scattering from parallel perfect electromagnetic conductor cylinders of circular cross-sections using an iterative procedure," Journal of Electromagnetic Waves and Applications, vol. 22, no. 7, pp. 987-1003, 2008.

[19] S. Ahmed and Q. A. Naqvi, "Electromagnetic scattering from a two dimensional perfect electromagnetic conductor (PEMC) strip and PEMC strip grating simulated by circular cylinders," Optics Communications, vol. 281, no. 17, pp. 4211-4218, 2008.

[20] V. G. Veselago, "The electrodynamics of substances with simultaneously negative values of $\varepsilon$ and $\mu$," Soviet Physics Uspekhi, vol. 10, no. 4, pp. 509-514, 1968.

[21] Z. Shen and C. Li, "Electromagnetic scattering by a conducting cylinder coated with metamaterials," Progress in Electromagnetic Research, vol. 42, pp. 91-105, 2003.

[22] S. Ahmed and Q. A. Naqvi, "Electromagnetic scattering from a perfect electromagnetic conductor circular cylinder coated with a metamaterial having negative permittivity and/or permeability," Optics Communications, vol. 281, no. 23, pp. 5664-5670, 2008.

[23] S. Ahmed and Q. A. Naqvi, "Electromagnetic scattering of two or more incident plane waves by a perfect electromagnetic conductor cylinder coated with a metamaterial having negative permitivity and/or permeability," Progress in Electromagnetic Research B, vol. 10, pp. 75-90, 2008.

[24] S. Ahmed and Q. A. Naqvi, "Directive em radiation of a line source in the presence of a coated nihility Cylinder," Journal of Electromagnetic Waves and Applications, vol. 23, no. 5, pp. 761-771, 2009. 
[25] S. Ahmed and Q. A. Naqvi, "Directive em radiation of a line source in the presence of a coated PEMC circular cylinder," Progress in Electromagnetics Research, vol. 92, pp. 91-102, 2009.

[26] S. Ahmed and Q. A. Naqvi, "Scattering of Electromagnetic Waves by a Coated Nihility Cylinder," Journal of Infrared, Millimeter, and Terahertz Waves, vol. 30, no. 10, pp. 1044-1052, 2009.

[27] R. F. Harrington, Time-Harmonic Electromagnetic Fields, chapter 5, McGraw-Hill, New York, NY, USA, 1961.

[28] W. Stutzman and G. Thiele, Antenna Theory and Design, chapter 9, John Wiley \& Sons, New York, NY, USA, 1981. 


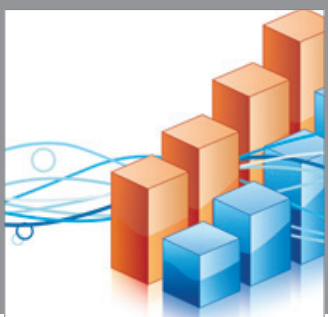

Advances in

Operations Research

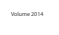

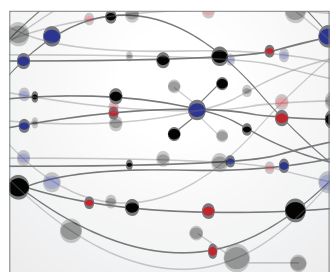

\section{The Scientific} World Journal
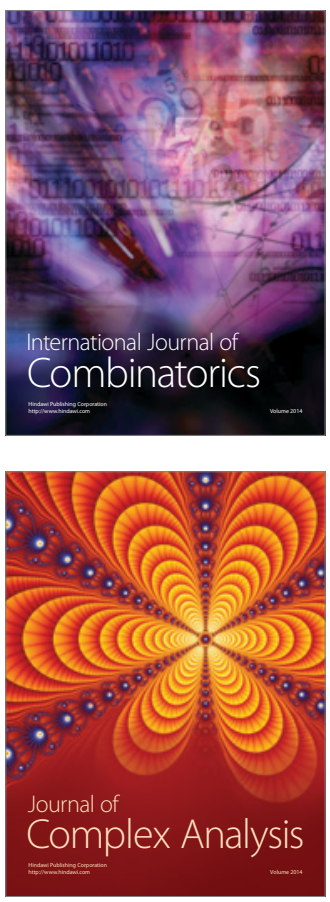

International Journal of

Mathematics and

Mathematical

Sciences
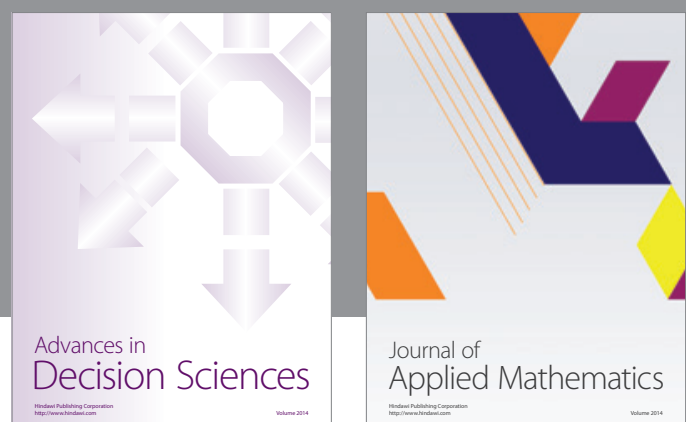

Journal of

Applied Mathematics
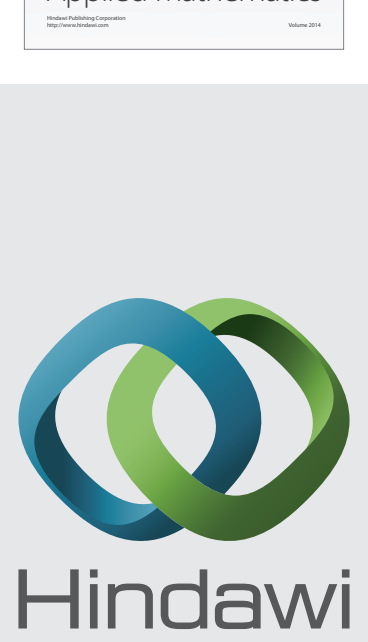

Submit your manuscripts at http://www.hindawi.com
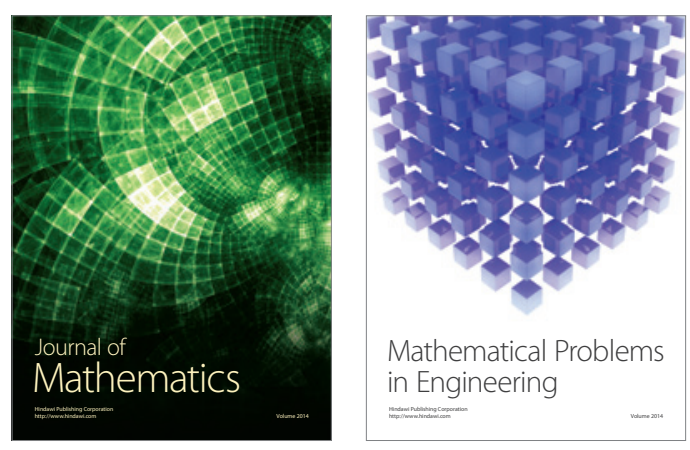

Mathematical Problems in Engineering
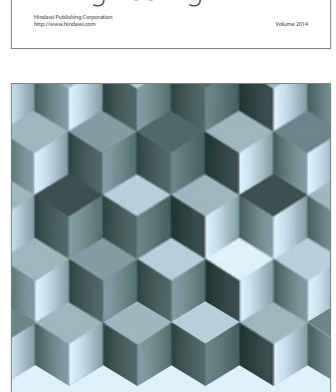

Journal of

Function Spaces
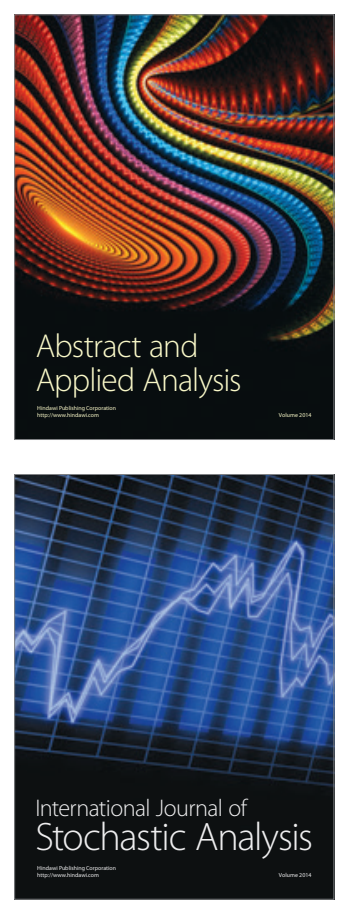

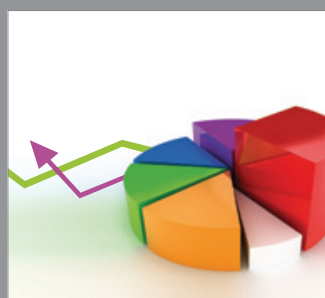

ournal of

Probability and Statistics

Promensencen
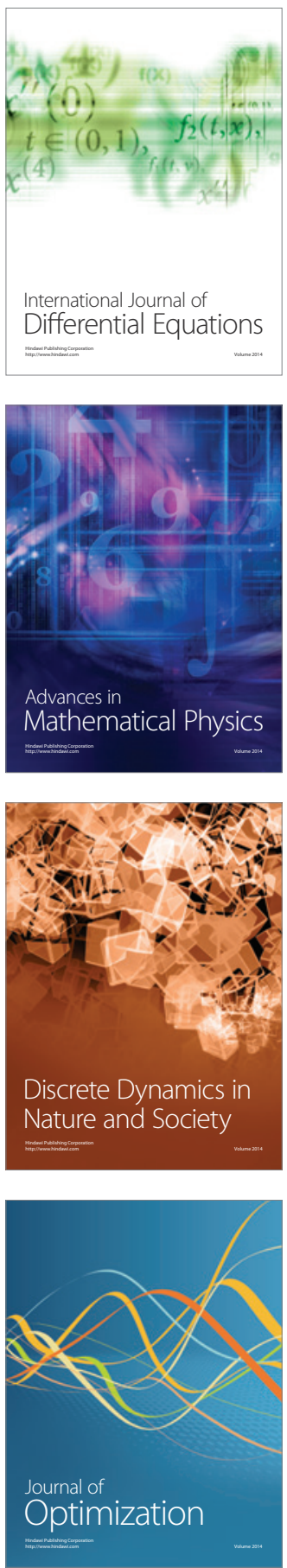